Research Article

\title{
A Novel Guidance Law for Intercepting a Highly Maneuvering Target
}

\author{
Gang Wu $(\mathbb{D}$ and Ke Zhang \\ School of Astronautics, Northwestern Polytechnical University, Xi'an 710072, China \\ Correspondence should be addressed to Gang Wu; wugang88@mail.nwpu.edu.cn
}

Received 14 September 2021; Revised 7 November 2021; Accepted 26 November 2021; Published 10 December 2021

Academic Editor: Shaoming He

Copyright (c) 2021 Gang Wu and Ke Zhang. This is an open access article distributed under the Creative Commons Attribution License, which permits unrestricted use, distribution, and reproduction in any medium, provided the original work is properly cited.

\begin{abstract}
Given the resolution of the guidance for intercepting highly maneuvering targets, a novel finite-time convergent guidance law is proposed, which takes the following conditions into consideration, including the impact angle constraint, the guidance command input saturation constraint, and the autopilot second-order dynamic characteristics. Firstly, based on the nonsingular terminal sliding mode control theory, a finite-time convergent nonsingular terminal sliding mode surface is designed. On the back of the backstepping control method, the virtual control law appears. A nonlinear first-order filter is constructed so as to address the "differential expansion" problem in traditional backstepping control. By designing an adaptive auxiliary system, the guidance command input saturation problem is dealt with. The RBF neural network disturbance observer is used for estimating the unknown boundary external disturbances of the guidance system caused by the target acceleration. The parameters of the RBF neural network are adjusted online in real time, for the purpose of improving the estimation accuracy of the RBF neural network disturbance observer and accelerating its convergence characteristics. At the same time, an adaptive law is designed to compensate the estimation error of the RBF neural network disturbance observer. Then, the Lyapunov stability theory is used to prove the finite-time stability of the guidance law. Finally, numerical simulations verify the effectiveness and superiority of the proposed guidance law.
\end{abstract}

\section{Introduction}

With the rapid development of space technology, the maneuverability of air strike weapons has become stronger and stronger, which has brought new challenges to interceptor missiles. Therefore, the success of intercepting highly maneuvering targets is drawing more and more attention [1]. In order to make the missile damage the target with maximum effectiveness, the missile not only needs to hit the target precisely but also needs to attack from the specified angle [2]. In addition, the convergence characteristics of the guidance law, the dynamic characteristics of the autopilot, and the input saturation constraints of the guidance commands are important factors for the successful interception of highly maneuvering targets. However, during the design process of the current guidance law, these factors are rarely considered comprehensively. As we all know, the more factors are pon- dered in the design of a guidance law, the more difficultly it will be designed.

It is well-known that the proportional navigation guidance law (PNGL) has been widely employed and researched for decades in terms of its advantages such as simple structure, robustness and practicability [3]. The guidance commands generated by the classic proportional guidance law (PNGL) are proportional to the line-of-sight (LOS) angular rate of the missile to target. It can ensure that the LOS angular rate converges to near zero and successfully intercept the target. However, the guidance system with the classic proportional navigation guidance law (PNGL) cannot satisfy the interceptor to attack the target from the specified angle. In [4], a new biased proportional guidance law is proposed by adding a supplementary time-varying bias to the traditional proportional guidance law towards achieving the above effect. In [5], after the improvement of the traditional 
proportional guidance law, nonstationary and nonmaneuverable targets can be successfully intercepted at the desired impact angle. In [6], as for nonmaneuvering targets, a bias proportional guidance law is put forward, which takes into account the limitations of the seeker's look angle and acceleration capability. It is worth noting that the advanced form of the traditional proportional guidance law can meet the needs of the impact angle constraint, but it is aimed at the interception of nonmaneuvering targets. When it comes to intercepting highly maneuvering targets, the performance of the proportional navigation guidance law algorithm will be reduced. This defect promotes the research of the progressive guidance law algorithm.

With the aim of successfully intercepting maneuvering targets, many advanced modern robust guidance law algorithms have been employed, such as the nonlinear $H_{\infty}$ guidance law [7], the adaptive guidance law [8], the differential game guidance law [9], the $L_{2}$ gain guidance law [10], and the optimal guidance law [11]. Compared with the abovementioned robust guidance laws, the sliding mode guidance law $[12,13]$ appears as the most widespread one on account of its simple design process and good robustness to external disturbances [14]. However, the traditional sliding mode guidance law can only achieve asymptotic convergence in an infinite time, because the sliding mode manifold is a linear function [15]. Generally speaking, the flight time of missile terminal guidance is very short, so the design of guidance law should consider the finite-time convergence control method [16]. Additionally, the finite-time sliding mode control method has faster convergence and better robustness to model uncertainties and external disturbances. Inspired by this attractive characteristic, the finite-time stability sliding mode control has been applied in the speed and altitude control of air-breathing hypersonic vehicles [17], the attitude control of unmanned aerial vehicles [18], and spacecraft steering rendezvous [19]. Therefore, the sliding mode guidance law with finite-time convergence is worth further studying.

At present, certain sliding mode guidance laws that converge in a finite time have been reported. In [20], a novel sliding mode guidance law is put forward, which can guide the LOS angle and the LOS angular rate to zero or its small neighborhood in a finite time. In [21], based on the secondorder sliding mode control method, a finite-time convergence guidance law is proposed to achieve the requirements of accuracy, stability, and robustness. In [22], the issue of interceptor's impact angle control is discussed. Aiming at the impact angle problem, the guidance law is derived, which adopts the nonsingular terminal sliding mode and the finitetime convergence theory. However, in the above-mentioned research, the autopilot lag of the missile is ignored. During the process of real-word engineering practice, the autopilot lag problem always exists, which has a great influence on the guidance accuracy, especially while the target is maneuvering. This may lead to larger miss distances and even system instability. Hence, it is necessary to consider the dynamic characteristics of the autopilot in the guidance law design process. In [23], a new guidance law built on the second-order sliding mode considering impact angle constraint and autopilot lag is presented to intercept maneuvering targets with unknown acceleration. This guidance law treats the autopilot as first-order inertia. In fact, the missile's autopilot has high-order dynamic characteristics; therefore, it is more reasonable to approximate the missile's autopilot to the second-order dynamic characteristics. In [24], the guidance law considers the second-order dynamic characteristics of the missile autopilot, but it cannot converge in a finite time. In the process of the sliding mode control design, the system disturbance is usually eliminated by selecting the appropriate switching gain. In the sliding mode guidance law of intercepting maneuvering targets, the target maneuvering information is regarded as the external disturbance of the guidance system. The robustness of the sliding mode guidance law against the guidance system's external disturbance is also achieved by selecting the appropriate switching gain. To satisfy the arrival condition of the sliding mode surface, the switching gain should be selected to be greater than the upper bound of the external disturbance. Therefore, the sliding mode guidance law designed in reference [25] assumes that the upper bound of the target acceleration is known, while the target acceleration information cannot actually be known in advance. In order to solve the abovementioned problem, in [15], an improved adaptive sliding mode guidance law without the information of the upper bound of the target acceleration is introduced, where the upper bound of the target acceleration is estimated online by the designed adaptive law. However, using the sliding mode guidance law to achieve the target maneuvering robustness only by switching gain will bring serious chattering phenomenon to the guidance system.

In recent years, with the development of disturbance observer techniques, the sliding mode control and disturbance observer techniques are usually combined in the guidance system design. The techniques of disturbance and uncertain observers, such as nonlinear disturbance observer [26], the nonhomogeneous disturbance observer with finitetime convergence [2], the fixed-time convergence disturbance observer [27], the extended high gain observer [28], the robust nonlinear disturbance observer [29], the extended state observer [30-32], and nonlinear robust $\mathrm{H}$-infinity observers [33], combined with the sliding mode control, have made important contributions to the treatment of many uncertain disturbances and improve the robustness of the guidance system. However, the convergence rate of the aforementioned observers is mainly determined by the magnitude of the gains, and the transient process of the observers may adversely affect the performance of the guidance system.

Recently, the neural network [34, 35] has been widely applied in control systems because it can approximate any nonlinear function online with arbitrary precision to deal with the uncertainty of the system. Especially, the RBF neural network is the most widely used. In [36], the RBF neural network is used to accurately estimate the lumped disturbance of the system and to deal with the uncertainties of multi-AUV formation control model parameters and unknown current disturbances. In [37], the RBF neural network is used to approximate the nonlinearity of two- 
dimensional aeroelastic system and solve the model uncertainties in the system. So far, there are little literature about the RBF neural network applied to the guidance law design. With important theoretical and engineering application value, it applies the RBF neural network to the guidance system, eliminating the guidance system disturbance caused by the target maneuvering.

During the actual guidance law design, the missile's acceleration is limited. On condition that the guidance commands exceed the missile's usable acceleration range, the guidance system will have an acceleration saturation problem, which will cause serious deterioration of the guidance system's performance and even the system instability. So it is necessary to design a guidance law with antiacceleration saturation. Although the references [38, 39] consider the acceleration saturation constraint when designing the guidance law, they do not consider the missile autopilot dynamics characteristics or only consider the autopilot first-order dynamic characteristics, rather than the autopilot second-order dynamic characteristics that are closer to the actual situation. In [24], the acceleration saturation constraint and the missile autopilot secondorder dynamic characteristics are noticed, but this guidance law cannot converge in a finite time. It is of great significance to study a finite-time convergence guidance law that can accurately intercept highly maneuvering targets by simultaneously considering the missile autopilot second-order dynamic characteristics, acceleration saturation constraint, and impact angle constraint.

Motivated by the above analysis, a novel finite-time convergence guidance law for intercepting highly maneuvering targets is brought forward in this paper. The special contributions of this paper are as follows:

(1) Compared with existing impact angle constraint guidance laws $[1-3,16]$ etc., the proposed guidance law not only realizes the interception of highly maneuvering targets in a finite time but also is simultaneously in consideration of the autopilot's second-order dynamics characteristics, acceleration saturation constraint, and impact angle constraint

(2) An RBF neural network disturbance observer which can adaptively adjust parameters online in real time is advanced to estimate guidance system external disturbance

(3) Focusing on the problem of autopilot dynamic delay, the backstepping control method [40] is introduced into the guidance law design. For the traditional backstepping control methods, to avoid the problem of "differential expansion" caused by multiple derivatives of the virtual control variables, first-order linear low-pass filter [24] is usually used to obtain the derivative of the virtual control variables. However, the first-order linear low-pass filter cannot guarantee the system's finite-time convergence. For this reason, this paper proposes a nonlinear filter to guarantee the system's finite-time convergence
(4) An adaptive auxiliary system is proposed to solve the problem of guidance command input saturation in this paper. Compared with the antisaturation method of guidance command input proposed in reference [39], the method proposed in this paper is simpler in form and easier to implement in engineering.

The remained part of this article is organized as follows. Section 2 presents the relative motion equations of the missile and the target in a two-dimensional plane. In Section 3, based on the nonsingular terminal sliding mode control theory, the adaptive RBF neural network observer, and the backstepping control method, a novel finite-time convergence guidance law for intercepting a highly maneuvering target is come up with. The guidance law simultaneously considers the autopilot's second-order dynamics characteristics, acceleration saturation constraint, and impact angle constraint. Its finite-time stability is proved by the Lyapunov theory. The numerical simulation results are carried out in Section 4 to demonstrate the effectiveness and superiority of the proposed guidance law. In Section 5, the key conclusion of the whole paper is presented.

\section{Formulation of Guidance Mode}

The relative motion geometry of the missile interception target in the two-dimensional plane is displayed in Figure 1. In the figure, $M$ represents the center of mass of the missile, while $\mathrm{T}$ is the center of mass of the target. The relative motion equations of the missile and the target in a twodimensional plane can be obtained as shown in the following equations.

$$
\begin{gathered}
\dot{r}=V_{t} \cos \left(q-\theta_{t}\right)-V_{m} \cos \left(q-\theta_{m}\right), \\
r \dot{q}=-V_{t} \sin \left(q-\theta_{t}\right)+V_{m} \sin \left(q-\theta_{m}\right), \\
\dot{\theta}_{t}=\frac{a_{t}}{V_{t}}, \\
\dot{\theta}_{m}=\frac{a_{m}}{V_{m}},
\end{gathered}
$$

where $r$ is the relative distance between the missile and the target, $q$ is the LOS angle of the missile to the target, $V_{m}$ is the speed of the missile, $V_{t}$ is the speed of the target, $\theta_{m}$ is the direction angle of missile velocity, $\theta_{t}$ is the direction angle of target velocity, $a_{m}$ is the normal acceleration of the missile, and $a_{t}$ is the normal acceleration of the target.

Taking the derivative of Equation (2), the following is obtained.

$$
\begin{aligned}
\dot{r} \dot{q} & +r \ddot{q}=-\dot{q}\left[V_{t} \cos \left(q-\theta_{t}\right)-V_{m} \cos \left(q-\theta_{m}\right)\right] \\
& +V_{t} \dot{\theta}_{t} \cos \left(q-\theta_{t}\right)-V_{m} \dot{\theta}_{m} \cos \left(q-\theta_{m}\right)
\end{aligned}
$$

Combined with Equation (1), Equation (5) can be 


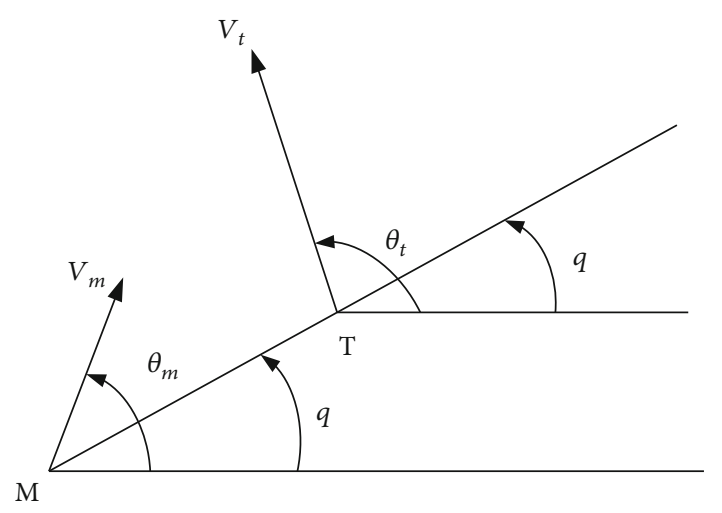

Figure 1: The relative motion geometry of the missile and the target.

arranged into the following.

$$
\ddot{q}=-\frac{2 \dot{r}}{r} \dot{q}-\frac{u_{q}}{r}+\frac{w_{q}}{r}
$$

where $u_{q}=a_{m} \cos \left(q-\theta_{m}\right)$ is the component of missile acceleration in the normal direction of the LOS of the missile to the target and $w_{q}=a_{t} \cos \left(q-\theta_{t}\right)$ is the component of the target acceleration in the normal direction of the LOS of the missile to the target.

Although the actual missile's autopilot has high-order dynamic characteristics, it can be approximated to the second-order dynamic characteristics in the guidance law design. The specific form is exhibited as follows.

$$
\ddot{u}_{q}=-2 \xi \omega_{n} \dot{u}_{q}-\omega_{n}^{2} u_{q}+\omega_{n}^{2} u,
$$

where $\xi$ is the damping ratio of the missile autopilot, $\omega_{n}$ is the natural frequency of the missile autopilot, and $u$ is the guidance command.

The actual missile autopilot has a saturation constraint on the input of the guidance command. After considering the saturation constraint of the guidance command, Equation (7) is rewritten as follows.

$$
\ddot{u}_{q}=-2 \xi \omega_{n} \dot{u}_{q}-\omega_{n}^{2} u_{q}+\omega_{n}^{2} \operatorname{sat}(u)
$$

where $\operatorname{sat}(\cdot)$ is the saturation function and $\operatorname{sat}(u)$ is expressed as

$$
\operatorname{sat}(u)=\left\{\begin{array}{lc}
u_{\max }, & u>u_{\max } \\
u, & -u_{\max } \leq u \leq u_{\max } \\
-u_{\max }, & u<-u_{\max }
\end{array}\right.
$$

where $u_{\max }$ is the upper bound of the guidance command.

Let $x_{1}=q-q_{d}, x_{2}=\dot{q}, x_{3}=u_{q}$, and $x_{4}=\dot{u}_{q}$, where $q_{d}$ is the expected terminal LOS angle. By combining Equations (6) and (8), the two-dimensional plane guidance equation that comprehensively considers the impact angle constraint, guidance command input saturation constraint, and autopi- lot second-order dynamic characteristics, which is expressed as

$$
\left\{\begin{array}{l}
\dot{x}_{1}=x_{2} \\
\dot{x}_{2}=a_{1} x_{2}+b_{1} x_{3}+d \\
\dot{x}_{3}=x_{4} \\
\dot{x}_{4}=a_{2} x_{4}+b_{2} x_{3}+b_{2} \operatorname{sat}(u),
\end{array}\right.
$$

where $a_{1}=-(2 \dot{r} / r), b_{1}=-(1 / r), a_{2}=-2 \xi \omega_{n}, b_{2}=\omega_{n}^{2}$, and $d$ $=w_{q} / r$.

\section{The Design of Guidance Law}

Before the guidance law design, in order to the convenience of guidance law design and finite-time convergence proof, the following lemmas are given:

Lemma 1 (see [41]). Assuming $x_{i}(i=1,2, \cdots, n)$ is an arbitrary real number, and there exists a real number $\alpha$ that satisfies $0<\alpha<1$, so that inequality (11) holds.

$$
\left(\sum_{i=1}^{n}\left|x_{i}\right|\right)^{\alpha} \leq \sum_{i=1}^{n}\left|x_{i}\right|^{\alpha} .
$$

Lemma 2 (see [42]). Assuming $x_{i}(i=1,2, \cdots, n)$ is an arbitrary real number, and there exists a real number $\beta$ that satisfies $\beta>1$, so that inequality (12) holds.

$$
n^{1-\beta}\left(\sum_{i=1}^{n}\left|x_{i}\right|\right)^{\beta} \leq \sum_{i=1}^{n}\left|x_{i}\right|^{\beta} .
$$

Lemma 3 (see $[43,44])$. If the first derivative of the Lyapunov function $V(x)$ satisfies inequality

$$
\dot{V}(x) \leq-a V^{\alpha}(x)-b V^{\beta}(x),
$$

where $a>0, b>0,0<\alpha<1$, and $\beta>1$, then the system will converge to the origin in a finite time.

In addition, if the system has a small disturbance, the first derivative of Lyapunov function $V(x)$ will satisfy inequality

$$
\dot{V}(x) \leq-a V^{\alpha}(x)-b V^{\beta}(x)+\varepsilon,
$$

where $\varepsilon$ is a small positive number, then the system converges to the neighborhood near the origin.

$$
\Omega=\left\{V \leq 2 v \mid a v^{\alpha}+b v^{\beta}=\varepsilon\right\} .
$$

Lemma 4 (see [45]). For the nonlinear system $\dot{x}=f(x, t), f($ $0, t)=0, x \in R^{n}$, if there is a continuously differentiable positive definite function $V$ and real numbers $c>0$ as well as 0 $<\alpha<1$, satisfying $\dot{V} \leq-c V^{\alpha}$, the system will converge to a 
stable equilibrium point origin in a finite time, and the convergence time $T$ satisfies $T \leq V^{1-\alpha}\left(x_{0}\right) / c(1-\alpha)$.

For the guidance system (10) taking into account the autopilot second-order dynamic characteristics and the guidance command saturation constraint, the missile can accurately intercept highly maneuvering targets at the desired impact angle. This paper combines the sliding mode control and backstepping ideas to design the guidance law. To make the system state converge in a finite time, the following nonsingular fast terminal sliding surface $s_{1}$ is designed as follows:

$$
s_{1}=x_{1}+k_{1} \operatorname{sig}\left(x_{1}\right)^{\alpha_{1}}+k_{2} \operatorname{sig}\left(x_{2}\right)^{\alpha_{2}},
$$

where $k_{1}>0, k_{2}>0, \alpha_{1}>\alpha_{2}$, and $1<\alpha_{2}<2$ are design parameters. $x_{1}$ and $x_{2}$ are the guidance system states, sig $\left(x_{i}\right)^{\alpha_{i}}=\left|x_{i}\right|^{\alpha_{i}} \operatorname{sgn}\left(x_{i}\right), i=1,2$.
In the following, the guidance law is designed by the backstepping method.

Step 1. Design the virtual control law of $x_{3}$.

The sliding mode error surface is $s_{2}$ defined as

$$
s_{2}=s_{1}=x_{1}+k_{1} \operatorname{sig}\left(x_{1}\right)^{\alpha_{1}}+k_{2} \operatorname{sig}\left(x_{2}\right)^{\alpha_{2}},
$$

where the definitions of parameters and variables are the same as that of Equation (16).

Combining the derivation of Equations (17) and (10), the following equation is obtained.

$$
\dot{s}_{2}=x_{2}+k_{1} \alpha_{1}\left|x_{1}\right|^{\alpha_{1}-1} x_{2}+k_{2} \alpha_{2}\left|x_{2}\right|^{\alpha_{2}-1}\left(a_{1} x_{2}+b_{1} x_{3}+d\right) \text {. }
$$

The virtual control law $x_{3 c}$ is designed as follows.

$$
x_{3 c}=-\frac{1}{b_{1}}\left[\frac{1}{k_{2} \alpha_{2}} \operatorname{sig}\left(x_{2}\right)^{2-\alpha_{2}}\left(1+k_{1} \alpha_{1}\left|x_{1}\right|^{\alpha_{1}-1}\right)+k_{3} \operatorname{sig}\left(s_{2}\right)^{\beta_{1}}+k_{4} \operatorname{sig}\left(s_{2}\right)^{\beta_{2}}+a_{1} x_{2}+\widehat{d}+\widehat{\delta}\right] \text {, }
$$

where $0<\beta_{1}<1, \beta_{2}>1, k_{3}>0$, and $k_{4}>0$ are design parameters. $\widehat{d}$ is the estimated value of the guidance system disturbance $d$ caused by the target maneuver. $\widehat{\delta}$ is an adaptive item which will be defined below. Other variables and parameters are the same as that of Equation (16).

For the purpose of achieving accurate interception of the highly maneuvering target, it is essential to accurately estimate the guidance system disturbance $d$ caused by target maneuvering. Therefore, this paper puts forth a parameter adaptive RBF neural network disturbance observer, and the expression of the disturbance is expressed as

$$
\mathrm{d}=\sum_{j=1}^{\mathrm{m}} w_{\mathrm{j}}^{*} \mathrm{~h}_{\mathrm{j}}^{*}=W^{* T} \mathrm{~h}^{*}
$$

where $\mathrm{W}^{*}=\left[\begin{array}{llll}w_{1}^{*} w_{2}^{*} & \cdots & w_{\mathrm{m}}^{*}\end{array}\right]^{T}$ is the optimal output weight vector of the output layer of the RBF neural network. $h^{*}=$ $\left[\begin{array}{lll}h_{1}^{*} h_{2}^{*} & \cdots & h_{\mathrm{m}}^{*}\end{array}\right]^{T}$ is the optimal vector whose elements are shown as the following equation, which represents the Gaussian activation function of the hidden layer neurons of the RBF neural network.

$$
\mathrm{h}_{\mathrm{j}}^{*}=\exp \left(\frac{-\left\|x-c_{\mathrm{j}}^{*}\right\|^{2}}{2 \sigma_{j}^{* 2}}\right), \mathrm{j}=1,2,3 \cdots m,
$$

where $c_{j}^{*}$ and $\sigma_{j}^{*}$, respectively, represent the optimal center and optimal width of the $\mathrm{j}_{\text {th }}$ Gaussian function. $x=[q, \dot{q}]$ is the input vector of the RBF neural network. $q$ and $\dot{q}$ are the LOS angle and the LOS angular rate of the missile to the target, respectively.

The parameter adaptive adjustment RBF neural network disturbance observer is expressed as

$$
\widehat{\mathrm{d}}=\sum_{j=1}^{\mathrm{m}} \widehat{w}_{\mathrm{j}} \widehat{\mathrm{h}}_{j}=W \wedge^{T} \widehat{\mathrm{h}}, \mathrm{j}=1,2,3 \cdots m,
$$

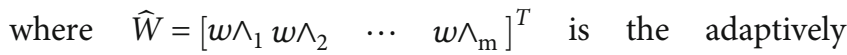
adjusted output weight vector of the $\mathrm{RBF}$ neural network output layer. $\widehat{h}=\left[\begin{array}{lll}h \wedge_{1} h \wedge_{2} & \cdots & h \wedge_{\mathrm{m}}\end{array}\right]^{T}$ is the Gaussian activation function vector of the RBF neural network hidden layer neurons, and its parameters can be adaptively adjusted. The elements of $\widehat{h}_{i}$ are expressed as

$$
\widehat{\mathrm{h}}_{\mathrm{j}}=\exp \left(\frac{-\left\|x-c \wedge_{j}\right\|^{2}}{2 \widehat{\sigma}_{j}^{2}}\right), \mathrm{j}=1,2,3 \cdots m
$$

where $\widehat{c}$ and $\widehat{\sigma}$, respectively, represent the adaptive center and adaptive width of the $\mathrm{j}_{\text {th }}$ Gaussian function. $x=[q, \dot{q}]$ is the input vector of the RBF neural network. $q$ and $\dot{q}$ are the LOS angle and the LOS angular rate of the missile to the target, respectively.

Let the disturbance $d$ subtract the estimation of the disturbance $\widehat{d}$. Then, the linearization method is used to expand it into a Taylor expansion series partially linear form. The 
following equation is obtained.

$\tilde{\mathrm{d}}=\mathrm{d}-\widehat{d}=W^{* T} \mathrm{~h}^{*}-W \wedge^{T} \widehat{\mathrm{h}}=-\tilde{W}^{T} \widehat{\mathrm{h}}-\widehat{W} \frac{\partial \mathrm{h}}{\partial c} \tilde{c}-\widehat{W} \frac{\partial \mathrm{h}}{\partial \sigma} \tilde{\sigma}+\varepsilon$,

where $\tilde{\mathrm{d}}$ is the disturbance estimation error of the RBF neural network. $\tilde{W}=\widehat{W}-\mathrm{W}^{*}$ is the weight vector deviation between the adaptive weight vector $\widehat{W}$ and the optimal weight vector $\mathrm{W}^{*}$ for the RBF neural network linear output layer. $\tilde{c}=\widehat{c}-c^{*}$ is the deviation center vector between the adaptive center vector $\widehat{c}_{i}$ and the optimal center vector $\mathrm{c}_{i}{ }^{*}$ for the Gaussian function. $\tilde{\sigma}=\widehat{\sigma}-\sigma^{*}$ is the deviation width vector between the adaptive width vector $\widehat{\sigma}$ and the optimal width vector $\sigma^{*} . \varepsilon$ is the error for the Taylor series. $\partial \mathrm{h} / \partial c$ and $\partial \mathrm{h} / \partial \sigma$ are, respectively, represented by the following equations.

$$
\begin{gathered}
\frac{\partial \mathrm{h}}{\partial c}=\mathrm{h}_{c}^{\prime}=\left[\begin{array}{cccc}
\frac{\partial \mathrm{h}_{1}}{\partial c_{1}} & 0 & 0 & 0 \\
0 & \frac{\partial \mathrm{h}_{2}}{\partial c_{2}} & 0 & 0 \\
\cdots & \cdots & \cdots & \cdots \\
\cdots & \cdots & \cdots & \frac{\partial \mathrm{h}_{m}}{\partial c_{m}}
\end{array}\right] \in R^{m \times m}, \\
\frac{\partial \mathrm{h}}{\partial \sigma}=\mathrm{h}_{\sigma}^{\prime}=\left[\begin{array}{cccc}
\frac{\partial \mathrm{h}_{1}}{\partial \sigma_{1}} & 0 & 0 & 0 \\
0 & \frac{\partial \mathrm{h}_{2}}{\partial \sigma_{2}} & 0 & 0 \\
\cdots & \cdots & \cdots & \cdots \\
\cdots & \cdots & \cdots & \frac{\partial \mathrm{h}_{m}}{\partial \sigma_{m}}
\end{array}\right] \in R^{m \times m} .
\end{gathered}
$$

Let $x_{3}=x_{3 c}+\varepsilon_{1}$, and substitute it into Equation (18). Thus, the following equation is obtained.

$$
\begin{aligned}
\dot{s}_{2}= & k_{2} \alpha_{2}\left|x_{2}\right|^{\alpha_{2}-1}\left(b_{1} \varepsilon_{1}+d-\widehat{d}-\widehat{\delta}\right)-k_{2} \alpha_{2}\left|x_{2}\right|^{\alpha_{2}-1} k_{3} \operatorname{sig}\left(s_{2}\right)^{\beta_{1}} \\
& -k_{2} \alpha_{2}\left|x_{2}\right|^{\alpha_{2}-1} k_{4} \operatorname{sig}\left(s_{2}\right)^{\beta_{2}} .
\end{aligned}
$$

Substitute Equation (24) into Equation (27), and the following equation is obtained.

$$
\begin{aligned}
\dot{s}_{2}= & k_{2} \alpha_{2}\left|x_{2}\right|^{\alpha_{2}-1}\left(-\tilde{W}^{T} \widehat{h}-\widehat{W} \frac{\partial h}{\partial c} \tilde{c}-\widehat{W} \frac{\partial h}{\partial \sigma} \tilde{\sigma}+\varepsilon+b_{1} \varepsilon_{1}-\widehat{\delta}\right) \\
& -k_{2} \alpha_{2}\left|x_{2}\right|^{\alpha_{2}-1} k_{3} \operatorname{sig}\left(s_{2}\right)^{\beta_{1}}-k_{2} \alpha_{2}\left|x_{2}\right|^{\alpha_{2}-1} k_{4} \operatorname{sig}\left(s_{2}\right)^{\beta_{2}} .
\end{aligned}
$$

Let $\delta=\varepsilon+b_{1} \varepsilon_{1}$ and $\tilde{\delta}=\widehat{\delta}-\delta$ and rewrite Equation (28) as

$$
\begin{aligned}
\dot{s}_{2}= & k_{2} \alpha_{2}\left|x_{2}\right|^{\alpha_{2}-1}\left(-\tilde{W}^{T} \widehat{h}-\widehat{W} \frac{\partial h}{\partial c} \tilde{c}-\widehat{W} \frac{\partial h}{\partial \sigma} \tilde{\sigma}-\tilde{\delta}\right) \\
& -k_{2} \alpha_{2}\left|x_{2}\right|^{\alpha_{2}-1} k_{3} \operatorname{sig}\left(s_{2}\right)^{\beta_{1}}-k_{2} \alpha_{2}\left|x_{2}\right|^{\alpha_{2}-1} k_{4} s i g\left(s_{2}\right)^{\beta_{2}} .
\end{aligned}
$$

The Lyapunov candidate function is selected as

$$
V_{0}=\frac{1}{2} s_{2}^{2}+\frac{1}{2 \eta_{1}} \tilde{\delta}^{2}+\frac{1}{2 \eta_{2}} \tilde{W}^{T} \tilde{W}+\frac{1}{2 \eta_{3}} \tilde{c}^{T} \tilde{c}+\frac{1}{2 \eta_{4}} \tilde{\sigma}^{T} \tilde{\sigma}
$$

where $\eta_{1}, \eta_{2}, \eta_{3}$, and $\eta_{4}$ are the parameters to be designed.

Take the derivative Lyapunov function, and the following equation is obtained.

$$
\dot{\mathrm{V}}_{0}=\mathrm{s}_{2} \dot{\mathrm{s}}_{2}+\frac{1}{\eta_{1}} \tilde{\delta} \tilde{\tilde{\delta}}+\frac{1}{\eta_{2}} \tilde{W}^{T} \dot{\tilde{W}}+\frac{1}{\eta_{3}} \tilde{c}^{T} \dot{\tilde{c}}+\frac{1}{\eta_{4}} \tilde{\sigma}^{T} \dot{\tilde{\sigma}}
$$

Substitute Equation (29) into Equation (31), and the following equation is obtained.

$$
\begin{aligned}
\dot{\mathrm{V}}_{0}= & \mathrm{s}_{2}\left[\begin{array}{l}
k_{2} \alpha_{2}\left|x_{2}\right|^{\alpha_{2}-1}\left(-\tilde{W}^{T} \widehat{\mathrm{h}}-\widehat{W} \frac{\partial \mathrm{h}}{\partial c} \tilde{c}-\widehat{W} \frac{\partial \mathrm{h}}{\partial \sigma} \tilde{\sigma}-\tilde{\delta}\right)- \\
k_{2} \alpha_{2}\left|x_{2}\right|^{\alpha_{2}-1} k_{3} \operatorname{sig}\left(s_{2}\right)^{\beta_{1}}-k_{2} \alpha_{2}\left|x_{2}\right|^{\alpha_{2}-1} k_{4} s i g\left(s_{2}\right)^{\beta_{2}}
\end{array}\right] \\
& +\frac{1}{\eta_{1}} \tilde{\delta} \dot{\tilde{\delta}}+\frac{1}{\eta_{2}} \tilde{W}^{T} \dot{\tilde{W}}+\frac{1}{\eta_{3}} \tilde{c}^{T} \dot{\tilde{c}}+\frac{1}{\eta_{4}} \tilde{\sigma}^{T} \dot{\tilde{\sigma}} .
\end{aligned}
$$

Substitute $\dot{\tilde{\delta}}_{\mathrm{i}}=\dot{\widehat{\delta}}_{i}, \quad \dot{\tilde{W}}_{\mathrm{i}}=\dot{\hat{W}}_{\mathrm{i}}, \dot{\tilde{\mathrm{c}}}_{\mathrm{i}}=\dot{\hat{\mathrm{c}}}_{i}$, and $\dot{\tilde{\sigma}}_{\mathrm{i}}=\dot{\widehat{\sigma}}_{i}$ into Equation (32), and the following equation is obtained.

$$
\begin{aligned}
\dot{\mathrm{V}}_{0}= & s_{2}\left[\begin{array}{l}
k_{2} \alpha_{2}\left|x_{2}\right|^{\alpha_{2}-1}\left(-\tilde{W}^{T} \widehat{\mathrm{h}}-\widehat{W} \frac{\partial \mathrm{h}}{\partial c} \tilde{c}-\widehat{W} \frac{\partial \mathrm{h}}{\partial \sigma} \tilde{\sigma}-\tilde{\delta}\right)- \\
k_{2} \alpha_{2}\left|x_{2}\right|^{\alpha_{2}-1} k_{3} s i g\left(s_{2}\right)^{\beta_{1}}-k_{2} \alpha_{2}\left|x_{2}\right|^{\alpha_{2}-1} k_{4} s i g\left(s_{2}\right)^{\beta_{2}}
\end{array}\right] \\
& +\frac{1}{\eta_{1}} \tilde{\delta} \dot{\hat{\delta}}+\frac{1}{\eta_{2}} \tilde{W}^{T} \dot{\hat{W}}+\frac{1}{\eta_{3}} \tilde{c}^{T} \dot{\hat{c}}+\frac{1}{\eta_{4}} \tilde{\sigma}^{T} \dot{\hat{\sigma}} .
\end{aligned}
$$

Rearrange Equation (33), and the following equation is obtained.

$$
\begin{aligned}
\dot{\mathrm{V}}_{0}= & s_{2}\left(-k_{2} \alpha_{2}\left|x_{2}\right|^{\alpha_{2}-1} k_{3} s i g\left(s_{2}\right)^{\beta_{1}}-k_{2} \alpha_{2}\left|x_{2}\right|^{\alpha_{2}-1} k_{4} s i g\left(s_{2}\right)^{\beta_{2}}\right) \\
& +\tilde{\delta}\left(\frac{1}{\eta_{1}} \dot{\widehat{\delta}}-k_{2} \alpha_{2}\left|x_{2}\right|^{\alpha_{2}-1} s_{2}\right)+\tilde{W}^{T}\left(\frac{1}{\eta_{2}} \dot{\hat{W}}-k_{2} \alpha_{2}\left|x_{2}\right|^{\alpha_{2}-1} \widehat{\mathrm{h}} s_{2}\right) \\
& +\tilde{c}^{T}\left(\frac{1}{\eta_{3}} \dot{\hat{\mathcal{C}}}-k_{2} \alpha_{2}\left|x_{2}\right|^{\alpha_{2}-1} \mathrm{~h}_{c}^{\prime} \mathrm{s}_{2} \widehat{W}\right)+\tilde{\sigma}^{T}\left(\frac{1}{\eta_{4}} \dot{\hat{\sigma}}-k_{2} \alpha_{2}\left|x_{2}\right|^{\alpha_{2}-1} \mathrm{~h}_{\sigma}^{\prime} \mathrm{s}_{2} \widehat{W}\right) .
\end{aligned}
$$

To ensure the first derivative of the Lyapunov function $\dot{\mathrm{V}}_{0}<0$, the adaptive parameters that change with time are 
selected as follows:

$$
\begin{gathered}
\dot{\widehat{\delta}}=\eta_{1} k_{2} \alpha_{2}\left|x_{2}\right|^{\alpha_{2}-1} \mathrm{~s}_{2}, \dot{\hat{W}}=\eta_{2} k_{2} \alpha_{2}\left|x_{2}\right|^{\alpha_{2}-1} \widehat{\mathrm{h}} s_{2}, \\
\dot{\hat{c}}=\eta_{3} k_{2} \alpha_{2}\left|x_{2}\right|^{\alpha_{2}-1} \mathrm{~h}_{c}^{\prime} \mathrm{s}_{2} \widehat{W}, \dot{\hat{\sigma}}=\eta_{4} k_{2} \alpha_{2}\left|x_{2}\right|^{\alpha_{2}-1} \mathrm{~h}_{\sigma}^{\prime} \mathrm{s}_{2} \widehat{W} .
\end{gathered}
$$

Remark 6. For the general disturbance observer, the performance of the disturbance observer is highly dependent on the parameters of the observer. Compared with the adaptive $\mathrm{RBF}$ disturbance observer proposed in this paper, the parameters of the observer can be adjusted to the optimal in real time, and the disturbance observer can obtain the optimal observation performance.

For fear of the problem of "differential expansion" caused by multiple derivatives of the virtual control variables and ensure the system finite-time convergence at the same time, the first-order nonlinear filter is designed as follows:

$$
\left\{\begin{array}{l}
\dot{x}_{3 d}=\frac{1}{\tau} \operatorname{sig}\left(x_{3 c}-x_{3 d}\right)^{\beta_{1}}+\frac{1}{\tau} \operatorname{sig}\left(x_{3 c}-x_{3 d}\right)^{\beta_{2}} \\
x_{3 d}(0)=x_{3 c}(0),
\end{array}\right.
$$

where $\tau>0,0<\beta_{1}<1$, and $\beta_{2}>1$ are the parameters to be designed. $x_{3 c}$ is the input of the first-order nonlinear filter. $x_{3 d}$ is the output of the first-order nonlinear filter.

Step 2. Design the virtual control law of $x_{4}$.

The sliding mode error surface $s_{3}$ is defined as Equation (37).

$$
s_{3}=x_{3}-x_{3 d} .
$$

Take the derivative of Equation (37), and combine it with Equation (10). Then the following equation is obtained.

$$
\dot{s}_{3}=\dot{x}_{3}-\dot{x}_{3 d}=x_{4}-\dot{x}_{3 d} \text {. }
$$

The virtual control law is designed as follows.

$$
x_{4 c}=-k_{2} \alpha_{2}\left|x_{2}\right|^{\alpha_{2}-1} b_{1} s_{2}-k_{5} \operatorname{sig}\left(s_{3}\right)^{\beta_{1}}-k_{6} \operatorname{sig}\left(s_{3}\right)^{\beta_{2}}+\dot{x}_{3 d} \text {. }
$$

To avoid the problem of "differential expansion" caused by multiple derivatives of the virtual control variables and ensure the system finite-time convergence, the first-order nonlinear filter is designed as follows:

$$
\left\{\begin{array}{l}
\dot{x}_{4 d}=\frac{1}{\tau} \operatorname{sig}\left(x_{4 c}-x_{4 d}\right)^{\beta_{1}}+\frac{1}{\tau} \operatorname{sig}\left(x_{4 c}-x_{4 d}\right)^{\beta_{2}} \\
x_{4 d}(0)=x_{4 c}(0),
\end{array}\right.
$$

where $\tau>0,0<\beta_{1}<1$, and $\beta_{2}>1$ are the parameters to be designed. $x_{4 c}$ is the input of the first-order nonlinear filter. $x_{4 d}$ is the output of the first-order nonlinear filter.
Step 3. Design the actual guidance law $u$.

The problem of guidance command input saturation is dealt with by designing an adaptive auxiliary system revealed as follows.

$$
\dot{\lambda}=-\rho \lambda+b_{2} \Delta u
$$

where $\lambda$ is the status of the auxiliary system, $\Delta u=\operatorname{sat}(u)-u$, and $\rho>0$ is the parameter to be designed.

The sliding mode error surface $s_{4}$ is defined as follows.

$$
s_{4}=x_{4}-x_{4 d}-\lambda
$$

Take the derivative of Equation (42) and combine it with Equation (10) as well as Equation (41). Therefore, the following equation is obtained.

$$
\dot{s}_{4}=a_{2} x_{4}-b_{2} x_{3}+b_{2} u+\rho \lambda-\dot{x}_{4 d} \text {. }
$$

The actual guidance law is designed as follows.

$$
u=\frac{1}{b_{2}}\left(-a_{2} x_{4}+b_{2} x_{3}-\rho \lambda+\dot{x}_{4 d}-s_{3}-k_{7} \operatorname{sig}\left(s_{4}\right)^{\beta_{1}}-k_{8} \operatorname{sig}\left(s_{4}\right)^{\beta_{2}}\right) .
$$

Theorem 7. For the guidance system (10) with guidance command input saturation constraints, under the action of the guidance law (44), the system state $x_{1}$ converges to the area $\left|x_{1}\right| \leq 2 \varepsilon_{2}$ in a finite time, and the system state $x_{2}$ also converges to the area $\left|x_{2}\right| \leq\left(\varepsilon_{2} / k_{2}\right)^{1 / \alpha_{2}}$ in a finite time, where $\varepsilon_{2}$ is a small positive number. In other words, the LOS angular rate $\dot{q}$ can converge to near zero in a finite time, and the LOS angle can converge to the desired LOS angle $q_{d}$ in a finite time.

Proof. The error of the virtual control law after the firstorder nonlinear filter is defined as follows.

$$
g_{i}=x_{i d}-x_{i c}, i=3,4 \text {. }
$$

Take the derivative of Equation (45), and combine Equation (36) as well as Equation (40). Thus, the following equation can be obtained.

$$
\dot{g}_{i}=\dot{x}_{i d}-\dot{x}_{i c}=-\frac{1}{\tau} \operatorname{sig}\left(g_{i}\right)^{\beta_{1}}-\frac{1}{\tau} \operatorname{sig}\left(g_{i}\right)^{\beta_{2}}-\dot{x}_{i c}, i=3,4,
$$

$$
g_{i} \dot{g}_{i}=-\frac{1}{\tau} g_{i} \operatorname{sig}\left(g_{i}\right)^{\beta_{1}}-\frac{1}{\tau} g_{i} \operatorname{sig}\left(g_{i}\right)^{\beta_{2}}-g_{i} \dot{x}_{i c}, i=3,4,
$$

According to the literature [46], there exists $\sigma_{i}>0$, which satisfies $\left|\dot{x}_{i c}\right| \leq \sigma_{i}$, so the following inequality can be 
obtained.

$$
g_{i} \dot{g}_{i} \leq-\frac{1}{\tau}\left|g_{i}\right|^{\beta_{1}+1}-\frac{1}{\tau}\left|g_{i}\right|^{\beta_{2}+1}+\frac{g_{i}^{2}+\sigma_{i}^{2}}{2} .
$$

Combining Equations (18), (37), and (45), the following equation can be obtained.

$$
\begin{aligned}
\dot{s}_{2}= & x_{2}+k_{1} \alpha_{1}\left|x_{1}\right|^{\alpha_{1}-1} x_{2}+k_{2} \alpha_{2}\left|x_{2}\right|^{\alpha_{2}-1}\left(a_{1} x_{2}+b_{1} x_{3 c}+b_{1} g_{3}+b_{1} s_{3}+d\right) \\
= & k_{2} \alpha_{2}\left|x_{2}\right|^{\alpha_{2}-1}\left(b_{1} g_{3}+b_{1} s_{3}+d-\widehat{d}-\widehat{\delta}\right)-k_{2} \alpha_{2}\left|x_{2}\right|^{\alpha_{2}-1} k_{3} s i g\left(s_{2}\right)^{\beta_{1}} \\
& -k_{2} \alpha_{2}\left|x_{2}\right|^{\alpha_{2}-1} k_{4} s i g\left(s_{2}\right)^{\beta_{2} .}
\end{aligned}
$$

Combining Equations (38), (42), and (44), the following equation can be obtained.

$$
\begin{aligned}
\dot{s}_{3}= & x_{4}-\dot{x}_{3 d}=s_{4}+g_{4}+\lambda-k_{2} \alpha_{2}\left|x_{2}\right|^{\alpha_{2}-1} b_{1} s_{2}-k_{5} s i g\left(s_{3}\right)^{\beta_{1}} \\
& -k_{6} s i g\left(s_{3}\right)^{\beta_{2}} .
\end{aligned}
$$

Combining Equations (43) and (44), the following equation can be obtained.

$$
\dot{s}_{4}=a_{2} x_{4}-b_{2} x_{3}+b_{2} u+\rho \lambda-\dot{x}_{4 d}=-s_{3}-k_{7} s i g\left(s_{4}\right)^{\beta_{1}}-k_{8} s i g\left(s_{4}\right)^{\beta_{2}} .
$$

The Lyapunov candidate function is constructed as follows.

$$
V=\frac{1}{2} \sum_{i=2}^{4} s_{i}^{2}+\frac{1}{2} \sum_{i=3}^{4} g_{i}^{2}
$$

After taking the derivative Lyapunov function, the following equation can be obtained.

$$
\begin{aligned}
\dot{V}= & \sum_{i=2}^{4} s_{i} \dot{s}_{i}+\sum_{i=3}^{4} g_{i} \dot{g}_{i} \\
= & s_{2}\left(k_{2} \alpha_{2}\left|x_{2}\right|^{\alpha_{2}-1}\left(b_{1} g_{3}+b_{1} s_{3}+\tilde{d}-\widehat{\delta}-k_{3} s i g\left(s_{2}\right)^{\beta_{1}}-k_{4} s i g\left(s_{2}\right)^{\beta_{2}}\right)\right. \\
& +s_{3}\left(s_{4}+g_{4}+\lambda-k_{2} \alpha_{2}\left|x_{2}\right|^{\alpha_{2}-1} b_{1} s_{2}-k_{5} s i g\left(s_{3}\right)^{\beta_{1}}-k_{6} s i g\left(s_{3}\right)^{\beta_{2}}\right) \\
& +s_{4}\left(-s_{3}-k_{7} s i g\left(s_{4}\right)^{\beta_{1}}-k_{8} s i g\left(s_{4}\right)^{\beta_{2}}\right)+g_{3} \dot{g}_{3}+g_{4} \dot{g}_{4} \\
= & s_{2} k_{2} \alpha_{2}\left|x_{2}\right|^{\alpha_{2}-1} b_{1} g_{3}+s_{2} k_{2} \alpha_{2}\left|x_{2}\right|^{\alpha_{2}-1} b_{1} s_{3} \\
& +s_{2} k_{2} \alpha_{2}\left|x_{2}\right|^{\alpha_{2}-1}(\tilde{d}-\widehat{\delta})+s_{3} s_{4}+s_{3} g_{4}+s_{3} \lambda \\
& -s_{3} k_{2} \alpha_{2}\left|x_{2}\right|^{\alpha_{2}-1} b_{1} s_{2}-s_{3} s_{4}-k_{2} \alpha_{2}\left|x_{2}\right|^{\alpha_{2}-1} k_{3}\left|s_{2}\right|^{\beta_{1}+1} \\
& -k_{2} \alpha_{2}\left|x_{2}\right|^{\alpha_{2}-1} k_{4}\left|s_{2}\right|^{\beta_{2}+1}-k_{5}\left|s_{3}\right|^{\beta_{1}+1}-k_{6}\left|s_{3}\right|^{\beta_{2}+1} \\
& -k_{7}\left|s_{4}\right|^{\beta_{1}+1}-k_{8}\left|s_{4}\right|^{\beta_{2}+1}+g_{3} \dot{g}_{3}+g_{4} \dot{g}_{4} \\
= & s_{2} k_{2} \alpha_{2}\left|x_{2}\right|^{\alpha_{2}-1} b_{1} g_{3}+s_{2} k_{2} \alpha_{2}\left|x_{2}\right|^{\alpha_{2}-1}(\tilde{d}-\widehat{\delta})+s_{3} g_{4}+s_{3} \lambda \\
& -k_{2} \alpha_{2}\left|x_{2}\right|^{\alpha_{2}-1} k_{3}\left|s_{2}\right|^{\beta_{1}+1}-k_{2} \alpha_{2}\left|x_{2}\right|^{\alpha_{2}-1} k_{4}\left|s_{2}\right|^{\beta_{2}+1} \\
& -k_{5}\left|s_{3}\right|^{\beta_{1}+1}-k_{6}\left|s_{3}\right|^{\beta_{2}+1}-k_{7}\left|s_{4}\right|^{\beta_{1}+1}-k_{8}\left|s_{4}\right|^{\beta_{2}+1}+g_{3} \dot{g}_{3}+g_{4} \dot{g}_{4} .
\end{aligned}
$$

Combining inequality (48) and equation (53), the following inequality can be obtained.

$$
\begin{aligned}
\dot{V} \leq & k_{2} \alpha_{2}\left|x_{2}\right|^{\alpha_{2}-1} b_{1} s_{2} g_{3}+k_{2} \alpha_{2}\left|x_{2}\right|^{\alpha_{2}-1} s_{2} \tilde{d}-k_{2} \alpha_{2}\left|x_{2}\right|^{\alpha_{2}-1} s_{2} \widehat{\delta} \\
& +s_{3} g_{4}+s_{3} \lambda-k_{2} \alpha_{2}\left|x_{2}\right|^{\alpha_{2}-1} k_{3}\left|s_{2}\right|^{\beta_{1}+1} \\
& -k_{2} \alpha_{2}\left|x_{2}\right|^{\alpha_{2}-1} k_{4}\left|s_{2}\right|^{\beta_{2}+1}-k_{5}\left|s_{3}\right|^{\beta_{1}+1}-k_{6}\left|s_{3}\right|^{\beta_{2}+1} \\
& -k_{7}\left|s_{4}\right|^{\beta_{1}+1}-k_{8}\left|s_{4}\right|^{\beta_{2}+1}-\frac{1}{\tau}\left|g_{3}\right|^{\beta_{1}+1}-\frac{1}{\tau}\left|g_{3}\right|^{\beta_{2}+1} \\
& +\frac{g_{3}^{2}}{2}+\frac{\sigma_{3}^{2}}{2}-\frac{1}{\tau}\left|g_{4}\right|^{\beta_{1}+1}-\frac{1}{\tau}\left|g_{4}\right|^{\beta_{2}+1}+\frac{g_{4}^{2}}{2}+\frac{\sigma_{4}^{2}}{2} .
\end{aligned}
$$

After rearranging inequality (54), the following inequality can be obtained.

$$
\begin{aligned}
\dot{V} \leq & -k_{2} \alpha_{2}\left|x_{2}\right|^{\alpha_{2}-1} k_{3}\left|s_{2}\right|^{\beta_{1}+1}-k_{2} \alpha_{2}\left|x_{2}\right|^{\alpha_{2}-1} k_{4}\left|s_{2}\right|^{\beta_{2}+1} \\
& -k_{5}\left|s_{3}\right|^{\beta_{1}+1}-k_{6}\left|s_{3}\right|^{\beta_{2}+1}-k_{7}\left|s_{4}\right|^{\beta_{1}+1}-k_{8}\left|s_{4}\right|^{\beta_{2}+1} \\
& -\frac{1}{\tau}\left|g_{3}\right|^{\beta_{1}+1}-\frac{1}{\tau}\left|g_{3}\right|^{\beta_{2}+1}-\frac{1}{\tau}\left|g_{4}\right|^{\beta_{1}+1}-\frac{1}{\tau}\left|g_{4}\right|^{\beta_{2}+1} \\
& +\frac{k_{2} \alpha_{2}\left|x_{2}\right|^{\alpha_{2}-1}\left(\left|b_{1}\right|+2\right)}{2} s_{2}^{2}+\frac{k_{2} \alpha_{2}\left|x_{2}\right|^{\alpha_{2}-1}\left|b_{1}\right|+1}{2} g_{3}^{2}+s_{3}^{2} \\
& +g_{4}^{2}+\frac{1}{2} \lambda^{2}+\frac{\sigma_{3}^{2}}{2}+\frac{\sigma_{4}^{2}}{2}+\frac{k_{2} \alpha_{2}\left|x_{2}\right|^{\alpha_{2}-1}}{2} \tilde{d}^{2}+\frac{k_{2} \alpha_{2}\left|x_{2}\right|^{\alpha_{2}-1}}{2} \delta \wedge^{2} .
\end{aligned}
$$

When $0<m_{1}<1, m_{2}>1$, and $p \geq 0$, the inequality $p^{m_{1}}$ $+p^{m_{2}} \geq p$ always holds, so the following inequality holds.

$$
-n_{1} p^{m_{1}}-n_{2} p^{m_{2}}+n_{3} p \leq-\left(n_{1}-n_{3}\right) p^{m_{1}}-\left(n_{2}-n_{3}\right) p^{m_{2}},
$$

where $n_{1}>0, n_{2}>0$, and $n_{3}>0$.

According to inequality (56), inequality (55) can be written as follows.

$$
\begin{aligned}
\dot{V} \leq & -k_{2} \alpha_{2}\left|x_{2}\right|^{\alpha_{2}-1}\left(k_{3}-\frac{\left(\left|b_{1}\right|+2\right)}{2}\right)\left(s_{2}^{2}\right)^{\frac{\beta_{1}+1}{2}} \\
& -k_{2} \alpha_{2}\left|x_{2}\right|^{\alpha_{2}-1}\left(k_{4}-\frac{\left(\left|b_{1}\right|+2\right)}{2}\right)\left(s_{2}^{2}\right)^{\frac{\beta_{2}+1}{2}}-\left(k_{5}-1\right)\left(s_{3}^{2}\right)^{\frac{\beta_{1}+1}{2}} \\
& -\left(k_{6}-1\right)\left(s_{3}^{2}\right)^{\frac{\beta_{2}+1}{2}}-k_{7}\left(s_{4}^{2}\right)^{\frac{\beta_{1}+1}{2}}-k_{8}\left(s_{4}^{2}\right)^{\frac{\beta_{2}+1}{2}} \\
& -\left(\frac{1}{\tau}-\frac{k_{2} \alpha_{2}\left|x_{2}\right|_{2}^{\alpha_{2}-1}\left|b_{1}\right|+1}{2}\right)\left(g_{3}^{2}\right)^{\frac{\beta_{1}+1}{2}} \\
& -\left(\frac{1}{\tau}-\frac{k_{2} \alpha_{2}\left|x_{2}\right|^{\alpha_{2}-1}\left|b_{1}\right|+1}{2}\right)\left(g_{3}^{2}\right)^{\frac{\beta_{2}+1}{2}}-\left(\frac{1}{\tau}-1\right)\left(g_{4}^{2}\right)^{\frac{\beta_{1}+1}{2}} \\
& -\left(\frac{1}{\tau}-1\right)\left(g_{4}^{2}\right)^{\frac{\beta_{2}+1}{2}}+\frac{1}{2} \lambda^{2}+\frac{\sigma_{3}^{2}}{2}+\frac{\sigma_{4}^{2}}{2}+\frac{k_{2} \alpha_{2}\left|x_{2}\right|^{\alpha_{2}-1}}{2} \tilde{d}^{2} \\
& +\frac{k_{2} \alpha_{2}\left|x_{2}\right|^{\alpha_{2}-1}}{2} \delta \wedge^{2} .
\end{aligned}
$$


Let

$$
\begin{aligned}
A= & \min \left\{k_{2} \alpha_{2}\left|x_{2}\right|^{\alpha_{2}-1}\left(k_{3}-\frac{\left(\left|b_{1}\right|+2\right)}{2}\right),\left(k_{5}-1\right), k_{7},\right. \\
& \left.\cdot\left(\frac{1}{\tau}-\frac{k_{2} \alpha_{2}\left|x_{2}\right|^{\alpha_{2}-1}\left|b_{1}\right|+1}{2}\right),\left(\frac{1}{\tau}-1\right)\right\} \\
B= & \min \left\{k_{2} \alpha_{2}\left|x_{2}\right|^{\alpha_{2}-1}\left(k_{4}-\frac{\left(\left|b_{1}\right|+2\right)}{2}\right),\left(k_{6}-1\right), k_{8},\right. \\
& \left.\cdot\left(\frac{1}{\tau}-\frac{k_{2} \alpha_{2}\left|x_{2}\right|^{\alpha_{2}-1}\left|b_{1}\right|+1}{2}\right),\left(\frac{1}{\tau}-1\right)\right\} \\
\sigma_{0}= & \frac{1}{2} \lambda^{2}+\frac{\sigma_{3}^{2}}{2}+\frac{\sigma_{4}^{2}}{2}+\frac{k_{2} \alpha_{2}\left|x_{2}\right|^{\alpha_{2}-1}}{2} \tilde{d}^{2}+\frac{k_{2} \alpha_{2}\left|x_{2}\right|^{\alpha_{2}-1}}{2} \delta \wedge^{2} .
\end{aligned}
$$

The appropriate guidance parameters can be selected to satisfy $A>0$ and $B>0$, and (59) can be rewritten as follows.

$$
\dot{V} \leq-A\left[\sum_{i=2}^{4}\left(s_{i}^{2}\right)^{\frac{\beta_{1}+1}{2}}+\sum_{i=3}^{4}\left(g_{i}^{2}\right)^{\frac{\beta_{1}+1}{2}}\right]-B\left[\sum_{i=2}^{4}\left(s_{i}^{2}\right)^{\frac{\beta_{2}+1}{2}}+\sum_{i=3}^{4}\left(g_{i}^{2}\right)^{\frac{\beta_{2}+1}{2}}\right]+\sigma_{0} .
$$

According to Lemmas 1 and 2, inequality (59) can be rewritten as follows.

$$
\begin{aligned}
\dot{V} \leq & -2^{\frac{\beta_{1}+1}{2}} A\left[\frac{1}{2} \sum_{i=2}^{4}\left(s_{i}^{2}\right)+\frac{1}{2} \sum_{i=3}^{4}\left(g_{i}^{2}\right)\right]^{\frac{\beta_{1}+1}{2}} \\
& -5^{\frac{1-\beta_{2}}{2}} 2^{\frac{\beta_{2}+1}{2}} B\left[\frac{1}{2} \sum_{i=2}^{4}\left(s_{i}^{2}\right)+\frac{1}{2} \sum_{i=3}^{4}\left(g_{i}^{2}\right)\right]^{\frac{\beta_{2}+1}{2}}+\sigma_{0} .
\end{aligned}
$$

Combining with Equation (52), inequality (60) can be rewritten as follows.

$$
\dot{V} \leq-C_{1} V^{\frac{\beta_{1}+1}{2}}-C_{2} V^{\frac{\beta_{2}+1}{2}}+\sigma_{0}
$$

where $C_{1}=2^{\left(\beta_{1}+1\right) / 2} A$ and $C_{2}=5^{\left(1-\beta_{2}\right) / 2} 2^{\left(\beta_{2}+1\right) / 2} B$.

According to Lemma 3, the system converges to a small neighborhood (62) near the origin in a finite time.

$$
\Omega=\left\{V \leq 2 v \mid C_{1} v^{\frac{\beta_{1}+1}{2}}+C_{2} v^{\frac{\beta_{2}+1}{2}}=\sigma_{0}\right\}
$$

According to Equation (62), $V$ converges to a small neighborhood near the origin in a finite time. Therefore, $s_{2}$ also converges in a finite time. It is possible to make $s_{2}$ arbitrarily be small by selecting reasonable guidance parameters. Supposed that $s_{2}$ converges to a smaller area $\left|s_{2}\right|<\varepsilon_{2}$ in a finite time, where $\varepsilon_{2}$ is a small positive number, after $s_{2}$ convergence, let $s_{2}=\mu_{2}$, and then $\left|\mu_{2}\right|<\varepsilon_{2}$, and combining with Equation (17), the following equation can be obtained.

$$
\mu_{2}=x_{1}+k_{1} \operatorname{sig}\left(x_{1}\right)^{\alpha_{1}}+k_{2} \operatorname{sig}\left(x_{2}\right)^{\alpha_{2}} \text {. }
$$

Rearrange Equation (63) as follows.

$$
x_{1}+k_{1} \operatorname{sig}\left(x_{1}\right)^{\alpha_{1}}+\left(k_{2}-\frac{\mu_{2}}{\operatorname{sig}\left(x_{2}\right)^{\alpha_{2}}}\right) \operatorname{sig}\left(x_{2}\right)^{\alpha_{2}}=0 \text {. }
$$

Let $k=k_{2}-\left(\mu_{2} / \operatorname{sig}\left(x_{2}\right)^{\alpha_{2}}\right)$, and then Equation (64) can be written as follows.

$$
x_{1}+k_{1} \operatorname{sig}\left(x_{1}\right)^{\alpha_{1}}+k \operatorname{sig}\left(x_{2}\right)^{\alpha_{2}}=0 .
$$

According to Equation (65), the following equation can be obtained.

$$
\operatorname{sign}\left(x_{1}\right)=-\operatorname{sign}\left(x_{2}\right),\left|x_{2}\right|=k^{-\frac{1}{\alpha_{2}}}\left(\left|x_{1}\right|+k_{1}\left|x_{1}\right|^{\alpha_{1}}\right)^{\frac{1}{\alpha_{2}}} \text {. }
$$

According to equation (66), the following equation can be obtained.

$$
x_{2}=\left|x_{2}\right| \operatorname{sign}\left(x_{2}\right)=k^{-\frac{1}{\alpha_{2}}}\left(\left|x_{1}\right|+k_{1}\left|x_{1}\right|^{\alpha_{1}}\right)^{\frac{1}{\alpha_{2}}} \operatorname{sign}\left(x_{2}\right) \text {. }
$$

The Lyapunov candidate function is selected as follows.

$$
V_{x}=\frac{1}{2} x_{1}^{2}
$$

After taking the derivative Lyapunov function, combining with Equations (10) and (67), the following equation can be obtained.

$$
\begin{aligned}
\dot{V}_{x} & =x_{1} x_{2}=x_{1} k^{-\frac{1}{\alpha_{2}}}\left(\left|x_{1}\right|+k_{1}\left|x_{1}\right|^{\alpha_{1}}\right)^{\frac{1}{\alpha_{2}}} \operatorname{sign}\left(x_{2}\right) \\
& =-\left|x_{1}\right| k^{-\frac{1}{\alpha_{2}}}\left(\left|x_{1}\right|+k_{1}\left|x_{1}\right|^{\alpha_{1}}\right)^{\frac{1}{\alpha_{2}}}=-k^{-\frac{1}{\alpha_{2}}}\left(\left|x_{1}\right|^{\alpha_{2}+1}+k_{1}\left|x_{1}\right|^{\alpha_{1}+\alpha_{2}}\right)^{\frac{1}{\alpha_{2}}} \\
& \leq-k^{-\frac{1}{\alpha_{2}}}\left|x_{1}\right|^{\frac{\alpha_{2}+1}{\alpha_{2}}}=-(\sqrt{2})^{\frac{\alpha_{2}+1}{\alpha_{2}}} k^{-\frac{1}{\alpha_{2}}} V_{x}^{\frac{\alpha_{2}+1}{2 \alpha_{2}}} .
\end{aligned}
$$

According to Lemma 4 , the system will converge to $k$ $\leq 0$ in a finite time, that is to say $k_{2}-\left(\mu_{2} / \operatorname{sig}\left(x_{2}\right)^{\alpha_{2}}\right) \leq 0$; then the following inequality holds.

$$
\left|x_{2}\right| \leq\left(\frac{\left|\mu_{2}\right|}{k_{2}}\right)^{\frac{1}{\alpha_{2}}} \leq\left(\frac{\varepsilon_{2}}{k_{2}}\right)^{\frac{1}{\alpha_{2}}} \text {. }
$$

According to Equation (63) and inequality (70), the convergence region of the system state can be obtained as shown in the following inequality.

$$
\left|x_{1}\right| \leq\left|x_{1}\right|+k_{1}\left|x_{1}\right|^{\alpha_{1}} \leq k_{2}\left|x_{2}\right|^{\alpha_{2}}+\left|\mu_{2}\right| \leq 2 \varepsilon_{2} \text {. }
$$

Thus, Theorem 7 is proved.

Remark 8. For the selection of guidance parameters, it is indispensable to ensure that $A>0$ and $B>0 . \alpha_{1}, \alpha_{2}, k_{1}$, 


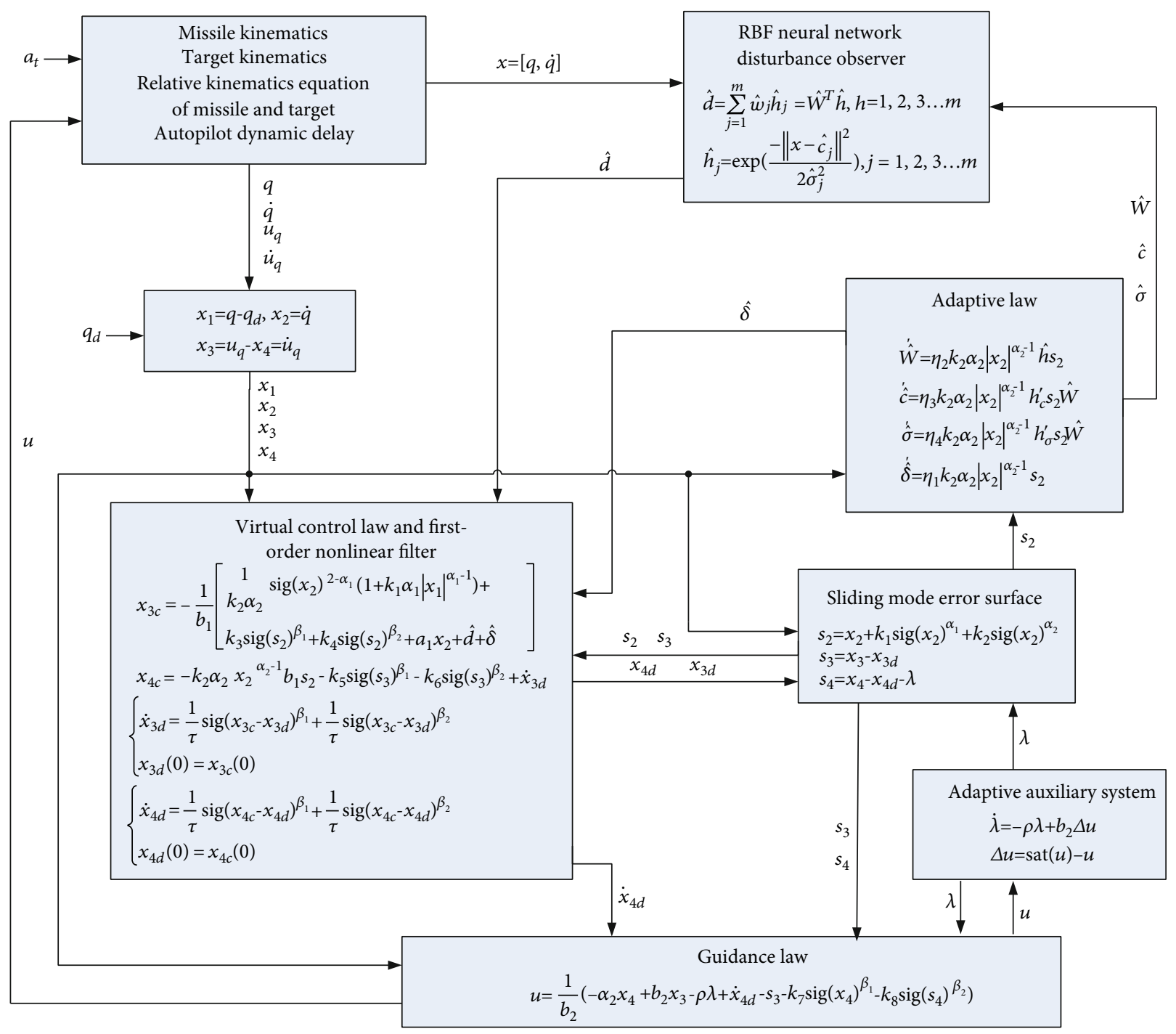

FIGURE 2: Block diagram of the proposed guidance law method.

and $k_{2}$ are selected according to the convergence speed required by the system states $x_{1}$ and $x_{2} . k_{3}, k_{4}, k_{5}, k_{6}, k_{7}$ , $k_{8}, \beta_{1}$, and $\beta_{2}$ are selected according to the required convergence speed of the sliding mode error surface $s_{2}, s_{3}$, and $s_{4} . \eta_{1}, \eta_{2}, \eta_{3}$, and $\eta_{4}$ determine the convergence speed of the adaptive RBF neural network. $\tau$ determines the filtering depth of the nonlinear filter. $\rho$ determines the convergence speed of the antisaturation adaptive auxiliary system.

The block diagram of the proposed guidance law method in this paper is shown in Figure 2.

\section{Simulation Results}

In this section, numerical simulation does duty for verifying the effectiveness and superiority of the guidance law intercepting highly maneuvering targets. Target maneuvers are divided into sinusoidal maneuvers and constant maneuvers. Numerical simulation is divided into two parts. In the first part, four missiles with different initial
TABLE 1: The initial condition of the missiles and expected terminal LOS angle.

\begin{tabular}{lccc}
\hline Missile & {$\left[x_{m}(0), y_{m}(0)\right](\mathrm{km})$} & {$\left[V_{x m}(0), V_{y m}(0)\right](\mathrm{m} / \mathrm{s})$} & $q_{d}\left(^{\circ}\right)$ \\
\hline M1 & {$[0,2]$} & {$[1200,0]$} & 20 \\
M2 & {$[3,10]$} & {$[1200,0]$} & 10 \\
M3 & {$[3,1]$} & {$[1200,0]$} & 25 \\
M4 & {$[10,0]$} & {$[1200,0]$} & 15 \\
\hline
\end{tabular}

states are used to intercept both sine and constant maneuvering targets with different expected terminal LOS angles, which verifies the terminal LOS angle control accuracy and target interception accuracy of the guidance law proposed in this paper. In the second part, different guidance laws are used to intercept sinusoidal and constant maneuvering targets under the same conditions so as to verify the superiority of the proposed guidance law in this paper compared with other guidance laws. 


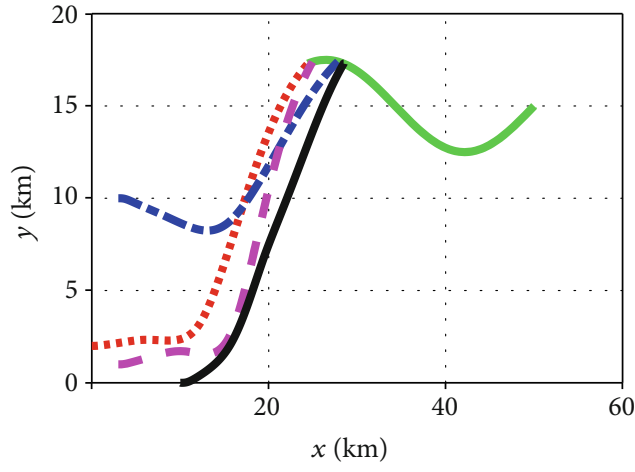

(a)
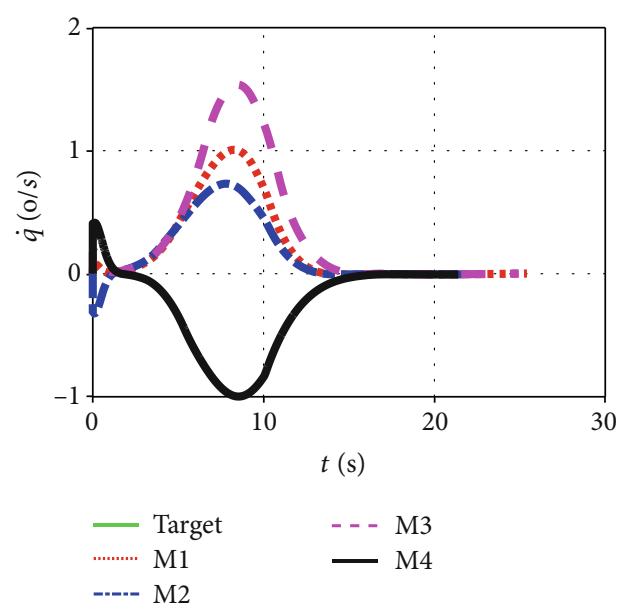

(c)

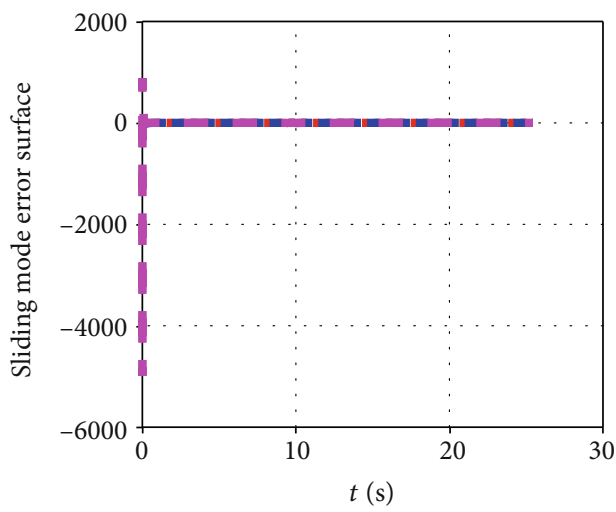

$\begin{array}{cc}\ldots . . . . . . . & s_{2} \\ ----- & s_{3} \\ --- & s_{4}\end{array}$

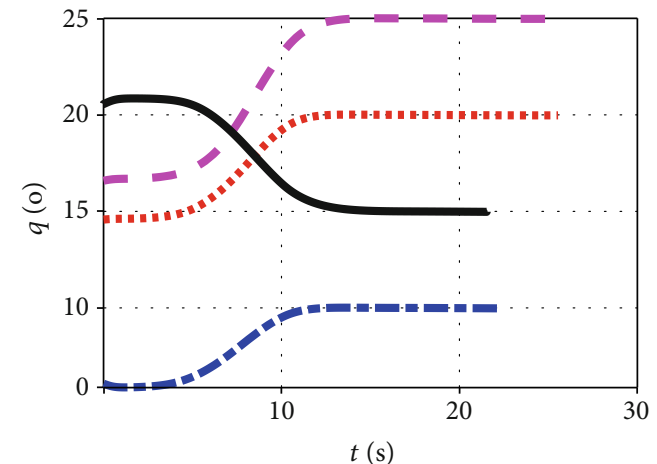

(b)

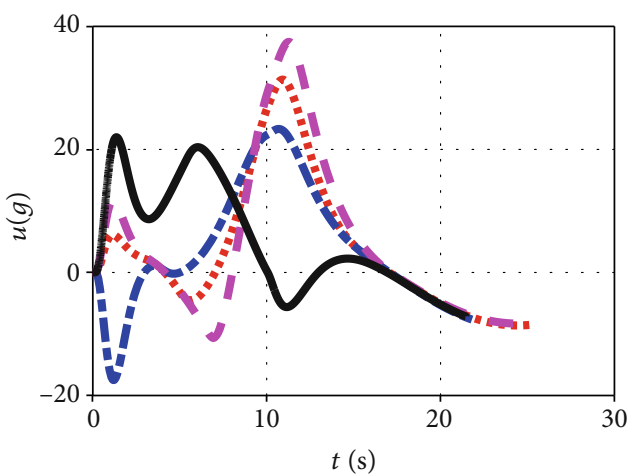

(d)

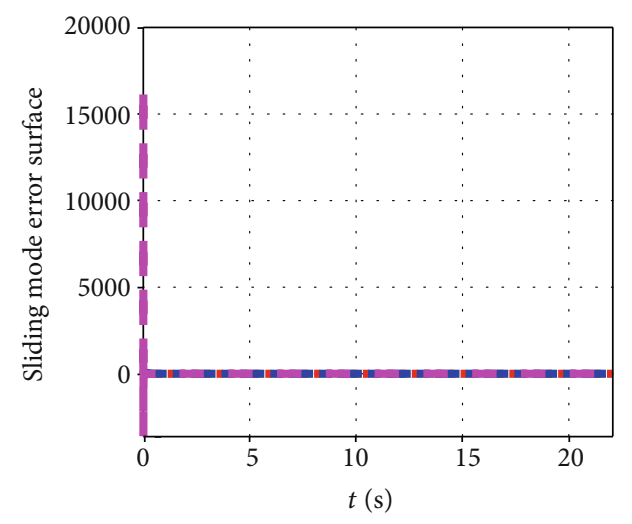

(f)

Figure 3: Continued. 


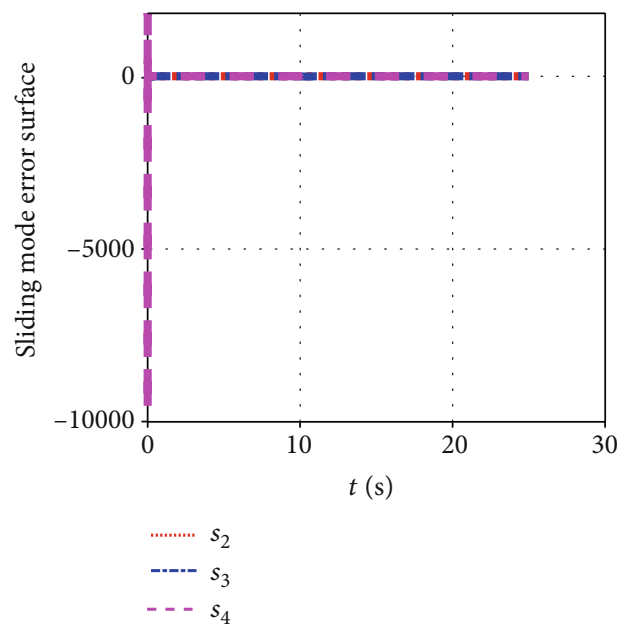

(g)

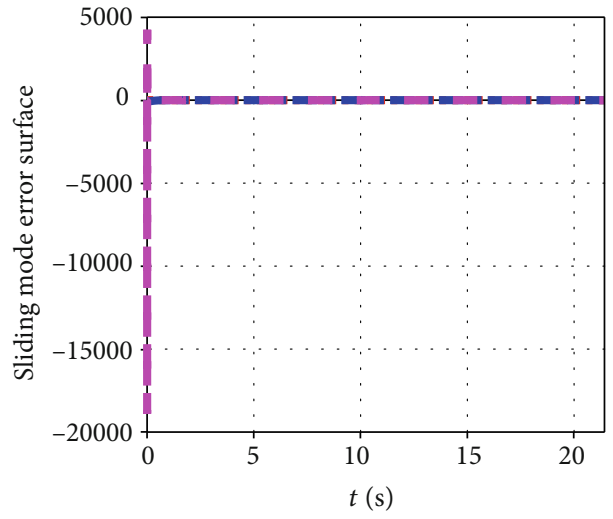

(h)

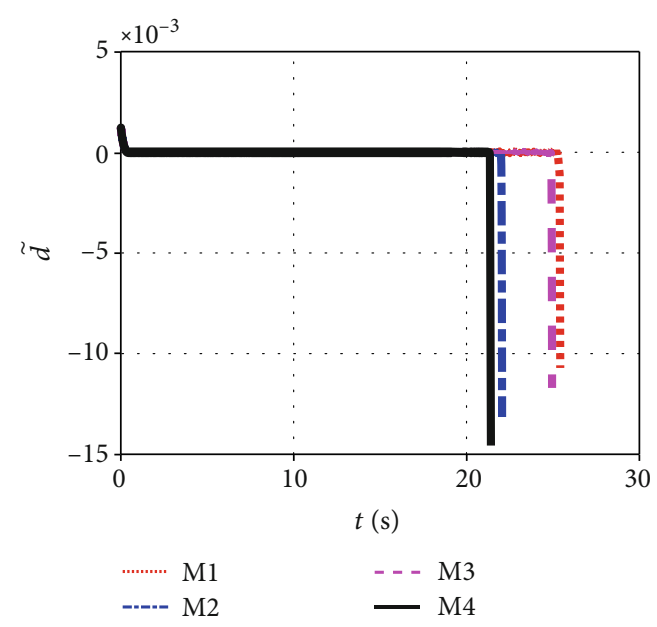

(i)

Figure 3: Simulation results of Case 1. (a) Relative motion curves of the missiles and targets. (b) Curves of $q$. (c) Curves of $\dot{q}$. (d) Curves of $u$. (e) Sliding mode error surface for M1. (f) Sliding mode error surface for M2. (g) Sliding mode error surface for M1. (h) Sliding mode error surface for M2. (i) Disturbance estimation error $\tilde{\mathrm{d}}$.

In the following simulation, the guidance law parameters proposed in this paper are set as follows:

$$
\begin{aligned}
\alpha_{1} & =3, \alpha_{2}=1.4, k_{1}=k_{2}=\min (20000 / \mathrm{r}, 40), k_{3}=k_{4} \\
& =2 a_{1}, k_{5}=k_{6}=k_{7}=k_{8}=4, \beta_{1}=0.5, \beta_{2}=1.9, \eta_{1}=\eta_{2} \\
& =\eta_{3}=\eta_{4}=0.1, \tau=0.01, \rho=10 .
\end{aligned}
$$

The initial values of the integral variable parameters in the guidance law are selected as follows:

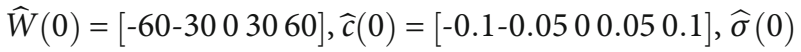

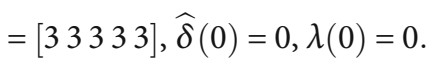

The second-order dynamic characteristic parameter of the missile autopilot is set as $w_{n}=10 \mathrm{rad} / \mathrm{s}, \xi=0.8$.
4.1. Different Missile Simulations. Four missiles in different initial states intercept sinusoidal and constant maneuvering targets with their respective expected terminal LOS angles. The initial state and expected terminal LOS angle setting of the missiles are represented in Table 1 . The target maneuvering case and its initial parameter settings are as follows:

Case 1. $a_{T}=100 \sin (0.2 t) \mathrm{m} / \mathrm{s}^{2}, x_{t}(0)=50 \mathrm{~km}, y_{t}(0)=15 \mathrm{~km}$, $V_{x t}(0)=-1000 \mathrm{~m} / \mathrm{s}$, and $V_{y t}(0)=-500 \mathrm{~m} / \mathrm{s}$.

Case 2. $a_{T}=100 \mathrm{~m} / \mathrm{s}^{2}, x_{t}(0)=50 \mathrm{~km}, y_{t}(0)=15 \mathrm{~km}, V_{x t}(0)$ $=-1000 \mathrm{~m} / \mathrm{s}$, and $V_{y t}(0)=-800 \mathrm{~m} / \mathrm{s}$.

Four missiles with different initial states and different expected terminal LOS angles intercepted two maneuvering targets in Case 1 and Case 2. The simulation results are shown in Figures 3 and 4 and Tables 2 and 3. Figures 3(a) and 4 (a) reveal the relative motion curves of the missile and the target. From the figures, it can be seen that the missiles with different initial states can successfully intercept the 


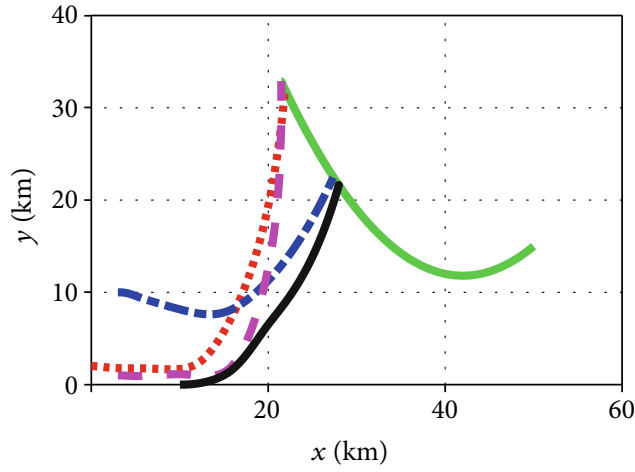

(a)
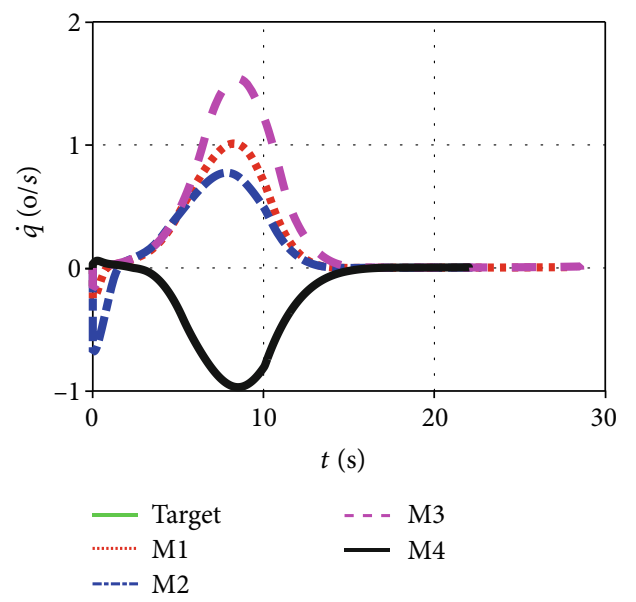

(c)

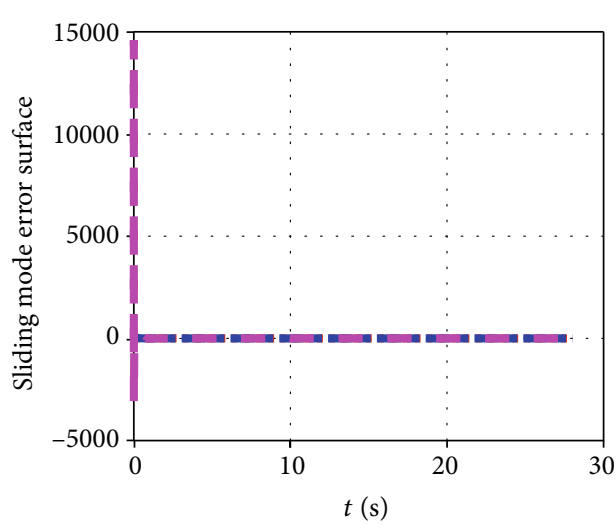

$\ldots \ldots \ldots \ldots s_{2}$

----- $s_{3}$

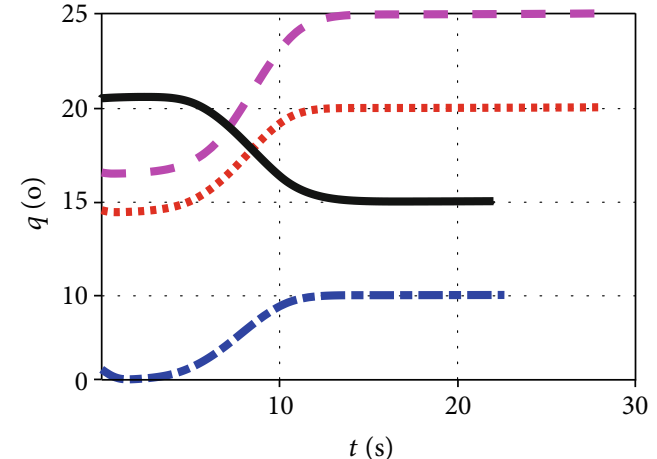

(b)

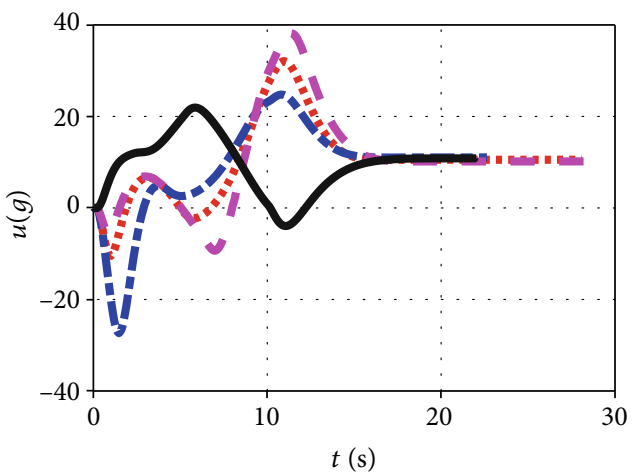

(d)

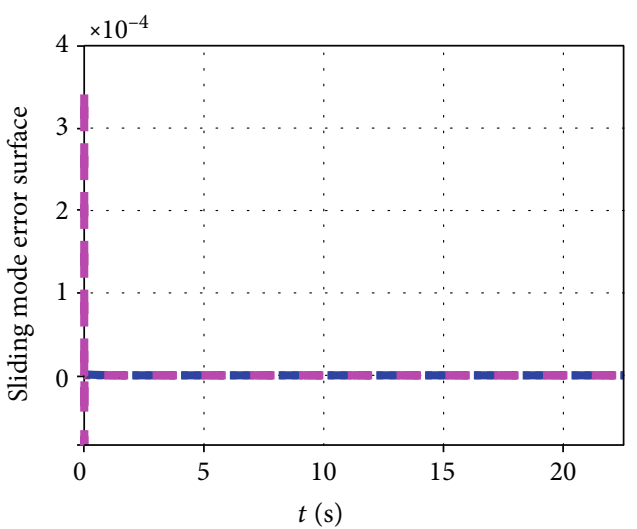

(f)

Figure 4: Continued. 


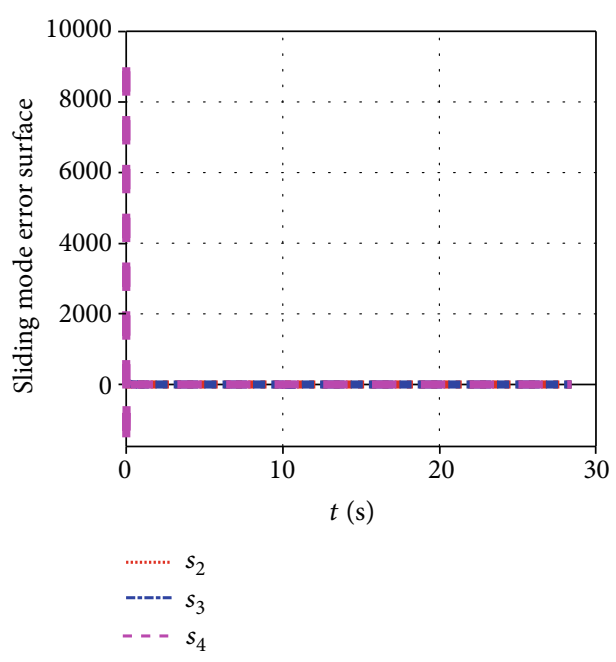

(g)

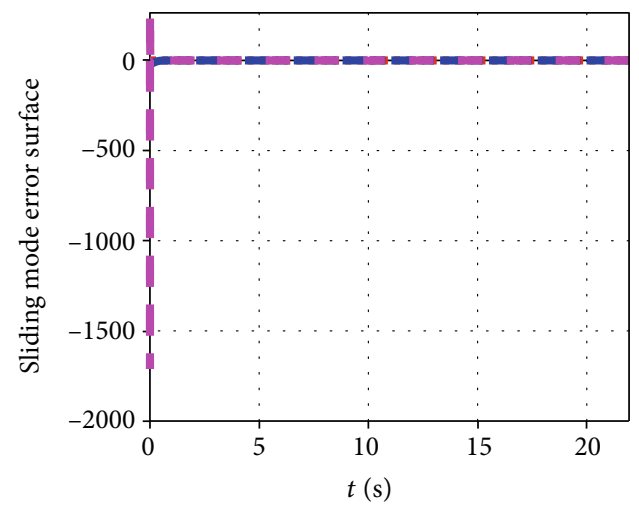

(h)

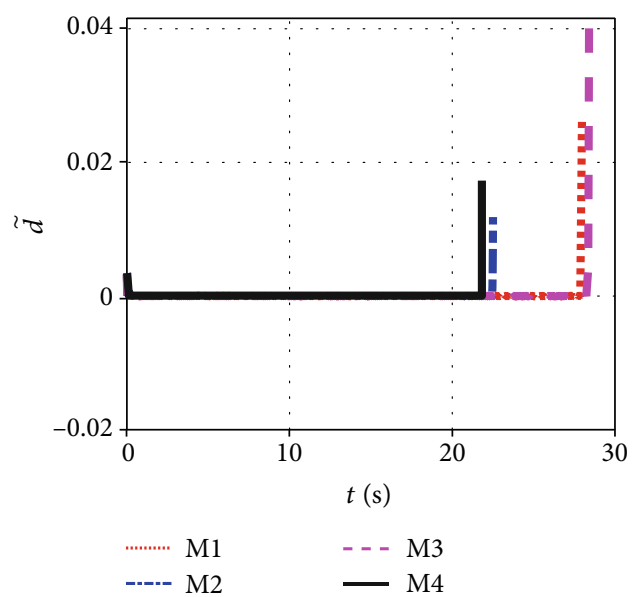

(i)

Figure 4: Simulation results of Case 2. (a) Relative motion curves of the missiles and targets. (b) Curves of $q$. (c) Curves of $\dot{q}$. (d) Curves of $u$. (e) Sliding mode error surface for M1. (f) Sliding mode error surface for M2. (g) Sliding mode error surface for M1. (h) Sliding mode error surface for M2. (i) Disturbance estimation error $\tilde{\mathrm{d}}$.

TABLE 2: Simulation results of Case 1.

\begin{tabular}{lccc}
\hline Missile & Miss distance $(\mathrm{m})$ & LOS angle error $\left(^{\circ}\right)$ & Flight time $(\mathrm{s})$ \\
\hline M1 & $6.3454 e-06$ & 0.0248 & 25.542 \\
M2 & $1.3616 e-04$ & 0.0298 & 22.177 \\
M3 & $4.1120 e-07$ & 0.0262 & 25.069 \\
M4 & $1.5057 e-04$ & 0.0514 & 21.532 \\
\hline
\end{tabular}

TABLe 3: Simulation results of Case 2.

\begin{tabular}{lccc}
\hline Missile & Miss distance $(\mathrm{m})$ & LOS angle error $\left(^{\circ}\right)$ & Flight time $(\mathrm{s})$ \\
\hline M1 & $5.0043 e-06$ & 0.074 & 28.104 \\
M2 & $6.2336 e-07$ & 0.0466 & 22.634 \\
M3 & $7.8147 e-07$ & 0.0926 & 28.54 \\
M4 & $2.6743 e-06$ & 0.057 & 22.025 \\
\hline
\end{tabular}

two maneuvering targets in Case 1 and Case 2. The curve of the LOS angle of the missile to the target is demonstrated in Figures 3(b) and 4(b). It can be clearly seen from the figures that although the initial state of the missile is different, for the two maneuvering targets in Case 1 and Case 2, the LOS angle of the missile to the target finally converged to the desired value with high accuracy. Figures 3(c) and 4(c) display the curves of the LOS angular rate. According to the figures, the LOS angular rate of four missiles in different initial states can rapidly converge to zero whether or not they intercept the maneuvering target in Case 1 or Case 2. Figures 3(d) and 4(d) indicate the guidance command curves. From the figures, it can tell by that the missiles with different initial states intercept two different maneuvering targets. The guidance commands calculated by the guidance law proposed in this paper are relatively smooth, and none of them exceed the range of amplitude. Figures 3(e)-3(h) and $4(\mathrm{e})-4(\mathrm{~h})$ show the sliding mode error surface curves of four missiles. All curves can converge to near zero. Figures 3(i) and 4(i) are disturbance estimation error curves. 


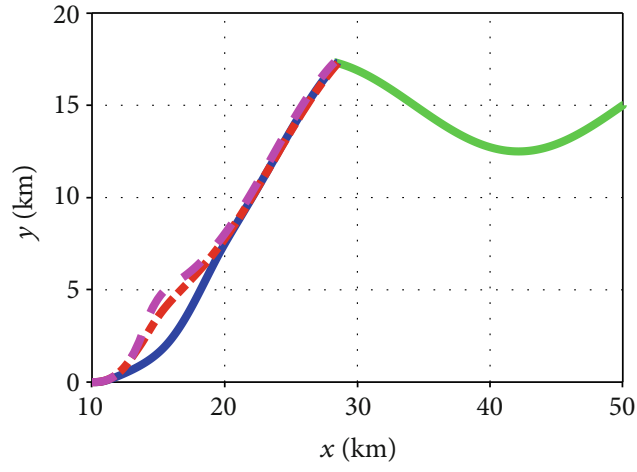

(a)

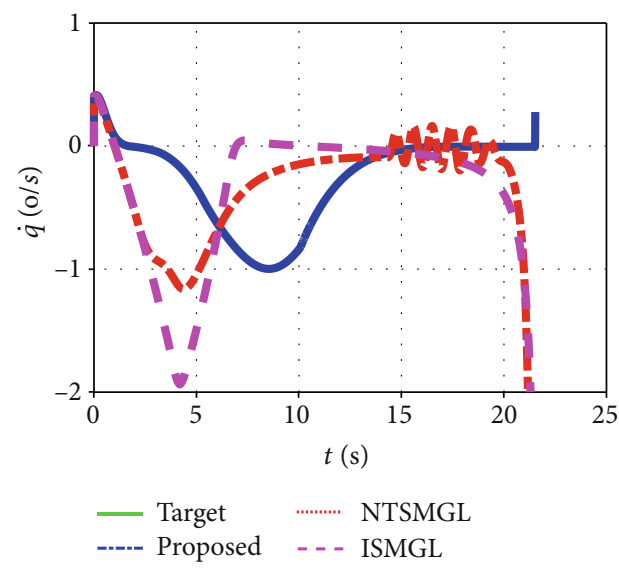

(c)

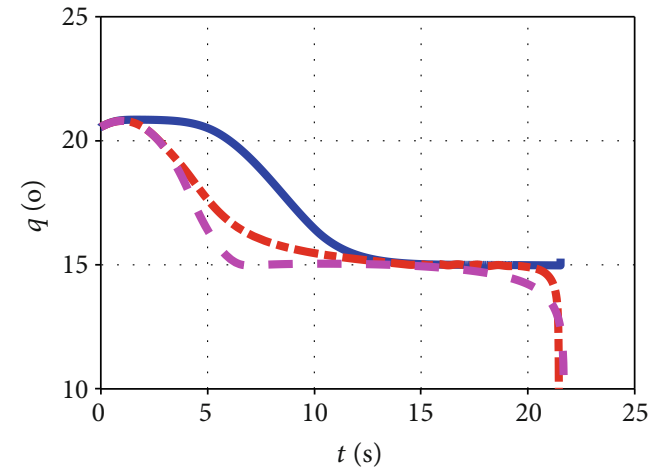

(b)

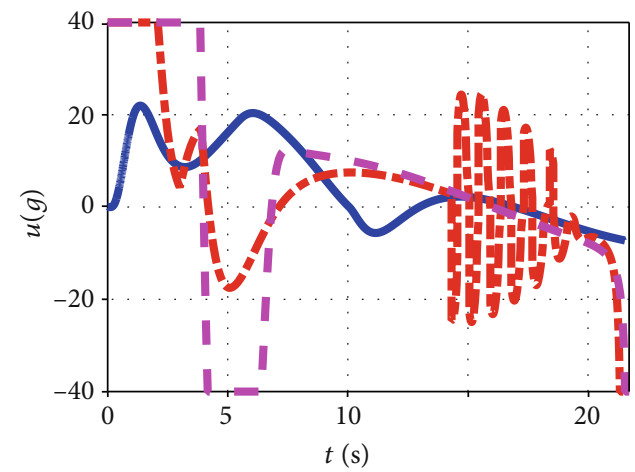

(d)

FIGURE 5: Simulation results of three different guidance laws in Case 1. (a) Relative motion curves of the missiles and targets. (b) Curves of $q$. (c) Curves of $\dot{q}$. (d) Curves of $u$.

From the figures, it can be clearly seen that the disturbance estimation error quickly converges to zero, although the disturbance caused by target maneuvering will increase rapidly at the moment before the missile and the target encounter, so will the disturbance estimation error. Tables 2 and 3 suggest the simulation results of four missiles in different initial states, which intercept the maneuvering targets in two cases with different expected terminal LOS angles. The maximum intercepted miss distance is $1.5057 e-04 \mathrm{~m}$, and the maximum terminal LOS angle error is $0.0926^{\circ}$, indicating that the guidance law proposed in this paper can control both the miss distance and the terminal LOS angle error with high accuracy. Therefore, the guidance law proposed in this paper can make missiles in different initial states successfully intercept different maneuvering targets with high accuracy, and the expected terminal LOS angle of the missile can be controlled with high accuracy.

4.2. Different Guidance Law Simulations. To confirm the superiority of the guidance law proposed in this paper, the simulation was compared with the nonlinear terminal sliding mode guidance law (NTSMGL) [47] and the integral sliding mode guidance law (ISMGL) [3]. Under the action of the three guidance laws, the initial parameters of the missile are set as $x_{m}(0)=10 \mathrm{~km}, y_{m}(0)=0 \mathrm{~km}, V_{x m}(0)=1200$ $\mathrm{m} / \mathrm{s}$, and $V_{y m}(0)=0 \mathrm{~m} / \mathrm{s}$, and the expected terminal LOS angle of the missile is set as $q_{d}=15^{\circ}$. Choose two different target maneuvering situations and target initial parameters in Case 1 and Case 2.

The sliding mode surface of the nonlinear terminal sliding mode guidance law (NTSMGL) is selected as follows.

$$
\mathrm{s}=x_{1}+\frac{1}{\beta} x_{2}^{p / q}
$$

where $\beta>0, p$, and $q$ are positive odd numbers, which satisfy $p>\mathrm{q}, 1<p / q<2$.

The nonlinear terminal sliding mode guidance law (NTSMGL) is expressed as follows.

$$
u_{N T S M G L}=r\left(-\frac{2 \dot{r}}{r} x_{2}+\frac{\beta \mathrm{q}}{p} x_{2}{ }^{2-p / q}+k s+h \operatorname{sgn}(s)\right) .
$$

The guidance parameter is selected as $\beta=10, p=9, \mathrm{q}=7$ , $\mathrm{k}=5.5$, and $h=10$.

The sliding mode surface of the integral sliding mode guidance law (ISMGL) is selected as follows.

$$
s=x_{2}+k_{2} x_{1}+k_{3} \int_{0}^{t}\left(k_{1} x_{2}+k_{2} x_{1}\right) d \tau \text {. }
$$




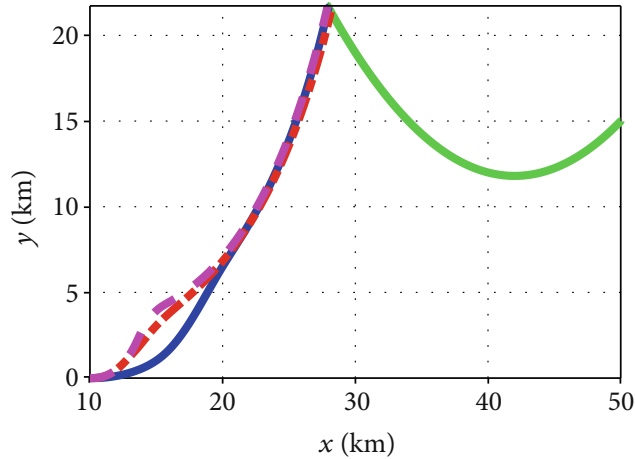

(a)

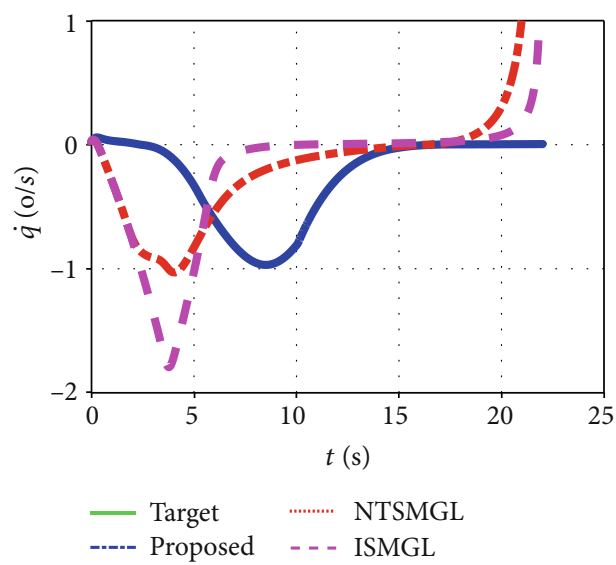

(c)

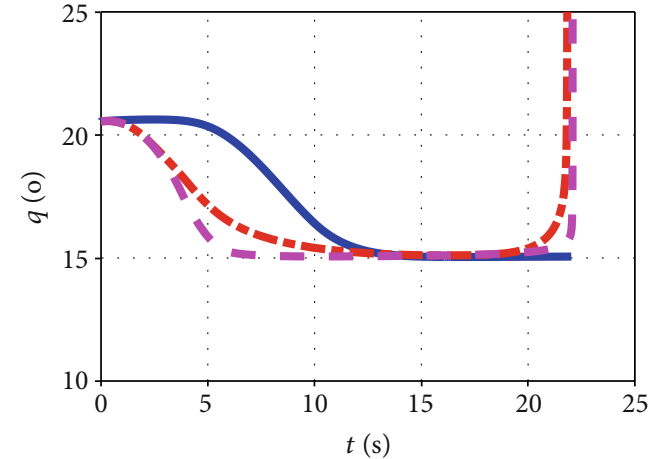

(b)

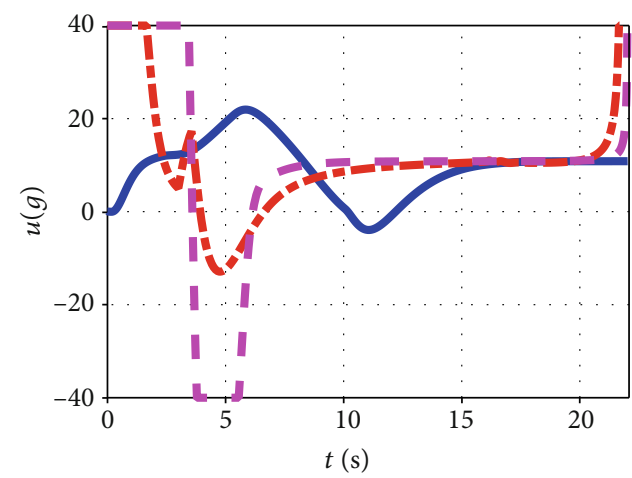

(d)

FiguRE 6: Simulation results of three different guidance laws in Case 2. (a) Relative motion curves of the missiles and targets. (b) Curves of $q$. (c) Curves of $\dot{q}$. (d) Curves of $u$.

The integral sliding mode guidance law (ISMGL) is expressed as follows.

$$
u=-2 \dot{r} x_{2}+r k_{2} x_{2}+r k_{3}\left(k_{1} x_{2}+k_{2} x_{1}\right)+\varepsilon \operatorname{sgn}(s) .
$$

The guidance parameter is selected as $k_{1}=1, k_{2}=1.5$, $k_{3}=1$, and $\varepsilon=70$.

For the sake of reducing the chattering caused by the discontinuous sign function in the nonlinear terminal sliding mode guidance law (NTSMGL) and the integral sliding mode guidance law (ISMGL), the sign function is replaced by the saturation function of Equation (78).

$$
\operatorname{sat}(s, \phi)=\left\{\begin{array}{l}
s / \phi,|s| \leq \phi \\
\operatorname{sgn}(s),|s|>\phi,
\end{array}\right.
$$

where $\phi=0.01$.

Three different guidance laws act on the missiles in the same initial state, with the same expected terminal LOS angle, intercepting the two maneuvering targets of Case 1 and Case 2. The simulation results are shown in Figures 5 and 6 and Tables 4 and 5. Figures 5(a) and 6(a) demonstrate the relative motion curves of missiles and targets. It can be seen from the figures that three different guidance laws acting on missiles in the same initial state can successfully
TABLE 4: Simulation results of three different guidance laws in Case 1.

\begin{tabular}{lccc}
\hline $\begin{array}{l}\text { Guidance } \\
\text { law }\end{array}$ & $\begin{array}{c}\text { Miss distance } \\
(\mathrm{m})\end{array}$ & $\begin{array}{c}\text { LOS angle error } \\
\left({ }^{\circ}\right)\end{array}$ & $\begin{array}{c}\text { Flight time } \\
(\mathrm{s})\end{array}$ \\
\hline Proposed & $1.5057 e-04$ & 0.0514 & 21.532 \\
NTSMGL & 2.0758 & 98.9872 & 21.467 \\
ISMGL & 2.8056 & 80.7839 & 21.719 \\
\hline
\end{tabular}

TABLE 5: Simulation results of three different guidance laws in Case 2.

\begin{tabular}{lccc}
\hline $\begin{array}{l}\text { Guidance } \\
\text { law }\end{array}$ & $\begin{array}{c}\text { Miss distance } \\
(\mathrm{m})\end{array}$ & $\begin{array}{c}\text { LOS angle error } \\
\left({ }^{\circ}\right)\end{array}$ & $\begin{array}{c}\text { Flight time } \\
(\mathrm{s})\end{array}$ \\
\hline Proposed & $2.6743 e-06$ & 0.057 & 22.025 \\
NTSMGL & 3.1417 & 61.6403 & 21.847 \\
ISMGL & 0.5615 & 64.0243 & 22.086 \\
\hline
\end{tabular}

intercept the maneuvering targets in Case 1 and Case 2, although the trajectories of the missiles are different. Under the action of three different guidance laws, the curve of the LOS angle of the missile to the target for two maneuvering targets in Case 1 and Case 2 is revealed by Figures 5(b) and $6(\mathrm{~b})$.The figures clearly demonstrate that the terminal 
LOS angle of the guidance law proposed in this paper is able to accurately converge to the desired value until the missile and the target encounter. Although the terminal LOS angles of the NTSMGL and ISMGL can also converge to the desired value, just a short time before the missile and the target encounter, the LOS angles of the two guidance laws have diverged to different degrees. Figures 5(c) and 6(c) show the curves of the LOS angular rate for two maneuvering targets in Case 1 and Case 2 under the action of three different guidance laws. It can be clearly seen from the figures that the guidance law proposed in this article can make the LOS angular rate accurately converge to near zero until the missile and the target encounter. Although the NTSMGL and the ISMGL can also make the LOS angular rate converge to near zero, just a short time before the missile and the target encounter, under the action of the two guidance laws, the LOS angular rate has a different degree of divergence phenomenon. Moreover, Figure 5(c) reveals that the NTSMGL has an oscillation phenomenon in the LOS angular rate from $15 \mathrm{~s}$ to $20 \mathrm{~s}$ for the maneuvering target in Case 1. Figures 5(d) and 6(d) demonstrate the missile's guidance command curves for two maneuvering targets in Case 1 and Case 2 under the action of three different guidance laws. As can be seen from the figures, in the guidance law proposed in this paper, the guidance command curve is relatively smooth throughout, and there is no command saturation in the whole process. Nevertheless, the NTSMGL and the ISMGL both present command saturation in the initial stage of guidance within a short period of time before the missile and the target encountered. Apart from this, it can be clearly seen from Figure 5(d) that for the maneuvering target in Case 1, and the guidance command of the NTSMGL appears an oscillation phenomenon from $15 \mathrm{~s}$ to $20 \mathrm{~s}$. Tables 4 and 5 demonstrate the simulation results of three different guidance laws, acting on missiles in the same initial state, and intercepting two maneuvering targets in Case 1 and Case 2, with the same expected terminal LOS angle. It can be seen from the table that the three different guidance laws have very little difference in interception time whether they are intercepting the maneuvering target in Case 1 or in Case 2. Three different guidance laws intercept the maneuvering targets in two cases. In terms of miss distance, the guidance law proposed in this paper has miss distances of $1.5057 e-04 \mathrm{~m}$ and $2.6743 e-06 \mathrm{~m}$, the NTSMGL has miss distances of $2.0758 \mathrm{~m}$ and $2.0758 \mathrm{~m}$, and the ISMGL has miss distances of $2.8056 \mathrm{~m}$ and $0.5615 \mathrm{~m}$. Consequently, the guidance law proposed in this paper is far less than the other two guidance laws in the miss distance. In terms of the LOS angle control, the guidance law proposed in this paper can control the LOS angle error in $0.0514^{\circ}$ and $0.057^{\circ}$, respectively, for maneuvering targets in two cases. However, the NTSMGL and the ISMGL had a divergence of the LOS angle for a short period of time before the missile and target encounter, and they were unable to control the LOS angle to the desired value. Therefore, the guidance law proposed in this paper has better control performance than the NTSMGL and the ISMGL in both miss distance and terminal LOS angle control.

\section{Conclusions}

In this paper, based on nonsingular terminal sliding mode control, the parameter adaptive adjustment RBF neural network disturbance observer, the finite-time stability theory, and the backstepping control method, a novel finite-time convergence guidance law that considers the attack angle constraint, the autopilot dynamic characteristics, and the guidance command saturation constraint are proposed. The main work and conclusions of this paper are as follows:

(1) To compensate for the disturbance of the guidance system caused by target maneuvering, an RBF neural network disturbance observer with parameters adaptive real-time online adjustment is put forward. At the same time, an adaptive law is designed to eliminate the estimation error of the RBF neural network disturbance observer

(2) Aiming at the problem of attacking angle constraint, a nonsingular terminal sliding mode surface with finite-time convergence is designed. In view of the dynamic delay of autopilot, a backstepping sliding mode guidance law with finite-time convergence is proposed. To solve the problem of guidance command input saturation, an adaptive auxiliary system is proposed

(3) Simulation results demonstrate that the proposed guidance law can make the missile accurately intercept the highly maneuvering target with the expected LOS angle for different missile initial conditions and expected LOS angle. The guidance law proposed in this paper has higher guidance accuracy and angle constraint accuracy than that of the existing guidance law.

For interception of highly maneuvering targets, the attack angle constraint, guidance command input saturation constraint, and dynamic characteristics of autopilot are comprehensively considered in the design of finite-time convergent guidance law. However, the antenna refraction error and measurement noise of the seeker are also important factors affecting the guidance accuracy, which are worthy of further consideration in the future research.

\section{Data Availability}

The data used to support the findings of this study are included within the article.

\section{Conflicts of Interest}

The authors declare that there is no conflict of interest regarding the publication of this paper.

\section{References}

[1] Y. J. Si and S. M. Song, "Continuous reaching law based threedimensional finite-time guidance law against maneuvering 
targets," Transactions of the Institute of Measurement and Control, vol. 41, no. 2, pp. 321-339, 2019.

[2] F. J. Zhao and H. You, "New three-dimensional second-order sliding mode guidance law with impact-angle constraints," Aeronautical Journal, vol. 124, no. 1273, pp. 368-384, 2020.

[3] W. J. Zhang, S. N. Fu, W. Li, and Q. L. Xia, “An impact angle constraint integral sliding mode guidance law for maneuvering targets interception," Journal of Systems Engineering and Electronics, vol. 31, no. 1, pp. 168-184, 2020.

[4] B. S. Kim, J. G. Lee, and H. S. Han, "Biased PNG law for impact with angular constraint," IEEE Transactions on Aerospace and Electronic Systems, vol. 34, no. 1, pp. 277-288, 1998.

[5] A. Ratnoo and D. Ghose, "Impact angle constrained guidance against nonstationary nonmaneuvering targets," Journal of Guidance Control and Dynamics, vol. 33, no. 1, pp. 269-275, 2010.

[6] B. G. Park, T. H. Kim, and M. J. Tahk, "Biased PNG with terminal-angle constraint for intercepting nonmaneuvering targets under physical constraints," IEEE Transactions on Aerospace and Electronic Systems, vol. 53, no. 3, pp. 15621572, 2017.

[7] C. D. Yang and H. Y. Chen, "Nonlinear H(infinity) robust guidance law for homing missiles," Journal of Guidance Control and Dynamics, vol. 21, no. 6, pp. 882-890, 1998.

[8] J. S. Huang, H. B. Zhang, G. J. Tang, and W. M. Bao, "Profiletracking-based adaptive guidance law against maneuvering targets," International Journal of Aerospace Engineering, vol. 2019, Article ID 8056342, 17 pages, 2019.

[9] R. Bardhan and D. Ghose, "Nonlinear differential games-based impact-angle-constrained guidance law," Journal of Guidance Control and Dynamics, vol. 38, no. 3, pp. 384-402, 2015.

[10] D. Zhou, C. D. Mu, and T. L. Shen, "Robust guidance law with L-2 gain performance," Transactions of the Japan Society for Aeronautical and Space Sciences, vol. 44, no. 144, pp. 82-88, 2001.

[11] T. N. Wang, S. J. Tang, J. Guo, and H. Q. Zhang, "Two-phase optimal guidance law considering impact angle constraint with bearings-only measurements," International Journal of Aerospace Engineering, vol. 2017, Article ID 1380531, 12 pages, 2017.

[12] H. G. Kim, D. Cho, and H. J. Kim, "Sliding mode guidance law for impact time control without explicit time-to-go estimation," IEEE Transactions on Aerospace and Electronic Systems, vol. 55, no. 1, pp. 236-250, 2019.

[13] Y. L. Wang, S. J. Tang, W. Shang, and J. Guo, “Adaptive fuzzy sliding mode guidance law considering available acceleration and autopilot dynamics," International Journal of Aerospace Engineering, vol. 2018, Article ID 6081801, 10 pages, 2018.

[14] J. H. Song and S. M. Song, "Robust impact angle constraints guidance law with autopilot lag and acceleration saturation consideration," Transactions of the Institute of Measurement and Control, vol. 41, no. 1, pp. 182-192, 2019.

[15] J. H. Song and S. M. Song, "Three-dimensional guidance law based on adaptive integral sliding mode control," Chinese Journal of Aeronautics, vol. 29, no. 1, pp. 202-214, 2016.

[16] P. Zhang and X. Y. Zhang, "A novel adaptive threedimensional finite-time guidance law with terminal angle constraints for interception of maneuvering targets," International Journal of Control, vol. 2, pp. 1-10, 2020.

[17] L. Q. Dou, M. M. Du, Q. Mao, and Q. Zong, "Finite-time nonsingular terminal sliding mode control-based fuzzy smooth- switching coordinate strategy for AHV-VGI," Aerospace Science and Technology, vol. 106, article 106080, 2020.

[18] R. J. Sun, Z. Zhou, and X. P. Zhu, "Flight quality characteristics and observer-based anti-windup finite-time terminal sliding mode attitude control of aileron-free full-wing configuration UAV," Aerospace Science and Technology, vol. 112, article 106638, 2021.

[19] S. A. Kasaeian, M. Ebrahimi, and N. Assadian, "Closed-loop powered-coast-powered predictive guidance for spacecraft rendezvous with non-singular terminal sliding mode steering," Acta Astronautica, vol. 166, pp. 507-523, 2020.

[20] K. B. Li, H. S. Shin, and A. Tsourdos, "Capturability of a sliding-mode guidance law with finite-time convergence," IEEE Transactions on Aerospace and Electronic Systems, vol. 56, no. 3, pp. 2312-2325, 2020.

[21] Y. Ji, D. F. Lin, W. Wang, and S. Y. Lin, "Finite-time convergent guidance law based on second-order sliding mode control theory," International Journal of Aeronautical and Space Sciences, vol. 18, no. 4, pp. 697-708, 2017.

[22] X. J. Zhang, M. Y. Liu, and Y. Li, "Nonsingular terminal sliding-mode-based guidance law design with impact angle constraints," Iranian Journal of Science and TechnologyTransactions of Electrical Engineering, vol. 43, no. 1, pp. 4754, 2019.

[23] W. J. Zhang, Q. L. Xia, and W. Li, "Novel second-order sliding mode guidance law with an impact angle constraint that considers autopilot lag for intercepting manoeuvering targets," Aeronautical Journal, vol. 124, no. 1279, pp. 1350-1370, 2020.

[24] S. T. Geng, J. Zhang, and J. G. Sun, “Adaptive back-stepping sliding mode guidance laws with autopilot dynamics and acceleration saturation consideration," Proceedings of the Institution of Mechanical Engineers Part G-Journal of Aerospace Engineering, vol. 233, no. 13, pp. 4853-4863, 2019.

[25] S. Sun, D. Zhou, and W. T. Hou, "A guidance law with finite time convergence accounting for autopilot lag," Aerospace Science and Technology, vol. 25, no. 1, pp. 132-137, 2013.

[26] N. Zhang, W. D. Gai, M. Y. Zhong, and J. Zhang, "A fast finitetime convergent guidance law with nonlinear disturbance observer for unmanned aerial vehicles collision avoidance," Aerospace Science and Technology, vol. 86, pp. 204-214, 2019.

[27] F. Yang and G. Xia, "A finite-time 3D guidance law based on fixed-time convergence disturbance observer," Chinese Journal of Aeronautics, vol. 33, no. 4, pp. 1299-1310, 2020.

[28] K. M. Ma, H. K. Khalil, and Y. Yao, "Guidance law implementation with performance recovery using an extended highgain observer," Aerospace Science and Technology, vol. 24, no. 1, pp. 177-186, 2013.

[29] D. Chwa, "Robust nonlinear disturbance observer based adaptive guidance law against uncertainties in missile dynamics and target maneuver," IEEE Transactions on Aerospace and Electronic Systems, vol. 54, no. 4, pp. 1739-1749, 2018.

[30] S. N. Gao, Z. H. Peng, D. Wang, and L. Liu, "Extended-stateobserver-based collision-free guidance law for target tracking of autonomous surface vehicles with unknown target dynamics," Complexity, vol. 2018, Article ID 4154670, 10 pages, 2018.

[31] E. J. Zhao, T. Chao, S. Y. Wang, and M. Yang, "Multiple flight vehicles cooperative guidance law based on extended state observer and finite time consensus theory," Journal of Aerospace Engineering, vol. 232, no. 2, pp. 270-279, 2018.

[32] M. J. Duan, D. Zhou, and D. L. Cheng, "Extended state observer-based finite-time guidance laws on account of 
thruster dynamics," Journal of Aerospace Engineering, vol. 233, no. 12, pp. 4583-4597, 2019.

[33] F. Liao, Q. Luo, H. B. Ji, and W. Gai, "Guidance laws with input saturation and nonlinear robust Ho observers," ISA Transactions, vol. 63, pp. 20-31, 2016.

[34] X. W. Bu and Q. Qi, "Fuzzy optimal tracking control of hypersonic flight vehicles via single-network adaptive critic design," IEEE Transactions on Fuzzy Systems, p. 1, 2020.

[35] X. W. Bu, Q. Qi, and B. Jiang, “A simplified finite-time fuzzy neural controller with prescribed performance applied to waverider aircraft," IEEE Transactions on Fuzzy Systems, vol. 99, pp. 1-9, 2021.

[36] H. B. Wang and B. Su, "Event-triggered formation control of AUVs with fixed-time RBF disturbance observer," Applied Ocean research, vol. 112, article 102638, 2021.

[37] J. X. Yuan, N. Qi, Z. Qiu, and F. X. Wang, "Adaptive RBF observer-sliding mode controller design for a two dimensional aeroelastic system with unsteady aerodynamics," Aerospace Science and Technology, vol. 80, pp. 482-495, 2018.

[38] D. F. Lin, Y. Ji, W. Wang, Y. C. Wang, H. Wang, and F. B. Zhang, "Three-dimensional impact angle-constrained adaptive guidance law considering autopilot lag and input saturation," International Journal of Robust and Nonlinear Control, vol. 30, no. 9, pp. 3653-3671, 2020.

[39] M. C. Ma, L. G. Tan, and S. M. Song, "Three-dimensional sliding mode guidance law for maneuvering target with prescribed performance and input saturation," Transactions of the Institute of Measurement and Control, vol. 43, no. 5, pp. 11761190, 2021.

[40] X. Bu, "Air-breathing hypersonic vehicles funnel control using neural approximation of non-affine dynamics," IEEE/ASME Transactions on Mechatronics, vol. 23, no. 5, pp. 2099-2108, 2018.

[41] G. Song, P. Shi, and R. K. Agarwal, "Fixed-time sliding mode cooperative control for multiagent networks via event- triggered strategy," International Journal of Robust and Nonlinear Control, vol. 31, no. 1, pp. 21-36, 2021.

[42] J. Xiao, Z. G. Zeng, A. L. Wu, and S. P. Wen, "Fixed-time synchronization of delayed Cohen-Grossberg neural networks based on a novel sliding mode," Neural Networks, vol. 128, pp. 1-12, 2020.

[43] L. A. Wang, H. B. Du, W. J. Zhang, D. Wu, and W. W. Zhu, "Implementation of integral fixed-time sliding mode controller for speed regulation of PMSM servo system," Nonlinear Dynamics, vol. 102, no. 1, pp. 185-196, 2020.

[44] X. Wang, J. Guo, S. J. Tang, and S. Qi, "Fixed-time disturbance observer based fixed-time back-stepping control for an airbreathing hypersonic vehicle," ISA Transactions, vol. 88, pp. 233-245, 2019.

[45] A. M. Zou, "Finite-time output feedback attitude tracking control for rigid spacecraft," IEEE Transactions on Control Systems Technology, vol. 22, no. 1, pp. 338-345, 2014.

[46] D. Swaroop, J. K. Hedrick, P. P. Yip, and J. C. Gerdes, "Dynamic surface control for a class of nonlinear systems," IEEE Transactions on Automatic Control, vol. 45, no. 10, pp. 1893-1899, 2000.

[47] S. R. Kumar and D. Ghose, "Three-dimensional impact angle guidance with coupled engagement dynamics," Journal of Aerospace Engineering, vol. 231, no. 4, pp. 621-641, 2017. 